\title{
Facilitation et simulation clinique : modalités de l'activité de guidage dans la situation simulée chez les formateurs en soins infirmiers
}

Facilitation and clinical simulation: Modalities of guidance by nurse trainers in simulation exercise

\section{Florence Policard}

\section{(2) OpenEdition}

\section{Journals}

Édition électronique

URL : http://journals.openedition.org/activites/3366

DOI : 10.4000/activites.3366

ISSN : $1765-2723$

\section{Éditeur}

ARPACT - Association Recherches et Pratiques sur les ACTivités

\section{Référence électronique}

Florence Policard, «Facilitation et simulation clinique : modalités de l'activité de guidage dans la situation simulée chez les formateurs en soins infirmiers », Activités [En ligne], 15-2 | 2018, mis en ligne le 15 octobre 2018, consulté le 19 avril 2019. URL : http://journals.openedition.org/activites/3366 ; DOI : 10.4000/activites.3366

Ce document a été généré automatiquement le 19 avril 2019

\section{cc) $(\mathrm{i})(\mathrm{\Theta}$}

Activités est mis à disposition selon les termes de la licence Creative Commons Attribution - Pas d'Utilisation Commerciale - Pas de Modification 4.0 International. 


\title{
Facilitation et simulation clinique : modalités de l'activité de guidage dans la situation simulée chez les formateurs en soins infirmiers
}

\author{
Facilitation and clinical simulation: Modalities of guidance by nurse trainers \\ in simulation exercise
}

Florence Policard

\section{NOTE DE L'ÉDITEUR}

Article soumis le 24 octobre 2017, accepté le 25 juin 2018

\section{Introduction}

1 La simulation clinique pleine échelle (SCPE) consiste à immerger des apprenants dans une situation de soin simulée et à les confronter à un problème à résoudre (par exemple prendre en charge un patient présentant un état clinique défaillant). Dans le débriefing qui suit la mise en situation, ils sont invités à s'interroger sur la manière dont ils ont géré ou cogéré le problème. Ce format pédagogique est de plus en plus utilisé dans la formation des professionnels de santé, en particulier en formation infirmière, celle-ci se réclamant d'une approche par les compétences. En 2009, la réforme de la formation a fait disparaitre la «mise en situation professionnelle» (MSP) support d'évaluation certificative en stage au profit d'une évaluation continue des compétences par les professionnels de terrain, rendant plus complexe l'objectivation des apprentissages professionnels des étudiants (ministère de la Santé et des Sports, 2009). Simultanément, la formation infirmière fait face à une convergence de facteurs défavorables tels que la pénurie de terrains de stage et le manque de disponibilité des professionnels de santé 
pour encadrer les étudiants (Delmas, \& St Pierre, 2011 ; Hetzell Campbell, \& Daley, 2009). Dans ce contexte, les pouvoirs publics envisagent la formation par la simulation en laboratoire comme une méthode complémentaire aux stages en milieu de soins (ministère des Affaires sociales et de la Santé, 2014). Ils l'inscrivent comme une "méthode prioritaire " devant prendre "une place de premier rang" (ministère des Affaires sociales et de la Santé, 2013), dans un mouvement général visant au développement des pédagogies actives. Elle est notamment recommandée pour travailler sur les problématiques de sécurité des soins et de qualité du travail en équipe (Granry, \& Moll, 2012). Son principal intérêt pédagogique réside dans un travail de réflexivité, à partir de la mise en activité des apprenants. C'est justement ce temps de mise à distance et de réflexion sur l'action qui manque aujourd'hui sur les terrains de stage. Il est question, au cours du débriefing, d'identifier des principes opératoires transférables à des situations futures analogues, réelles cette fois. La SCPE s'appuie en cela sur les principes de l'apprentissage expérientiel, de l'apprentissage par problème et de la conceptualisation dans l'action (Argyris, \& Schön, 1996 ; Kolb, 1984 ; Pastré, 2005) et vise la construction des compétences professionnelles.

2 Cet article s'intéresse à l'activité des formateurs dans ce format pédagogique, et plus particulièrement à leur activité de facilitation. La facilitation est encore peu étudiée dans les recherches sur la simulation. Sa définition n'est pas stabilisée: elle fait référence tantôt à un processus de médiation entre l'apprenant et le savoir au moment du débriefing, tantôt à une médiation entre l'apprenant et la situation au cours de l'exercice simulé. Cette seconde acception est assimilée à une fonction de guidage. Le guidage vise à faire évoluer les paramètres de la situation en réponse aux actions des apprenants et à orienter ces derniers en leur fournissant des indices pour qu'ils puissent réaliser l'activité cible. Le processus de guidage nous parait revêtir un enjeu important pour l'efficacité pédagogique de ces séances, car le matériau expérientiel produit en situation constitue le support du travail réflexif lors du débriefing.

3 Après avoir précisé les caractéristiques de la SCPE comme format pédagogique, nous replacerons la question du guidage dans l'activité de facilitation réalisée par le formateur. Puis nous recenserons les principaux apports de la recherche sur le guidage dans le contexte de la simulation. Enfin, nous tenterons de distinguer différentes modalités de guidage sur le couplage sujet-situation en cours de simulation clinique et d'identifier les intentions et les logiques qui sous-tendent ces modalités. Notre propos se fonde sur une recherche réalisée auprès de formateurs exerçant en institut de formation en soins infirmiers (Ifsi).

\section{Le format pédagogique «simulation clinique pleine échelle » et l'activité de facilitation}

4 L'utilisation de la simulation dans les formations de santé n'est pas une nouveauté, mais l'usage de simulateurs haute-fidélité et la reconstitution d'environnements d'apprentissage les plus fidèles possible à la réalité permettent d'envisager une autre approche pédagogique. Si les premières expériences de simulation pleine échelle reposaient sur une approche comportementale et sur une pédagogie de la répétition, la simulation clinique met aujourd'hui le débriefing sur le devant de la scène, considérant ce dernier comme l'étape clé dans la construction des apprentissages (Dreifuerst, 2012; 
Jeffries, 2005 ; Rudolph, Simon, Rivard, Dufresne, \& Reamer, 2007 ; Savoldelli, \& Boët, 2013). Il ne s'agit pas seulement d'acquérir des habiletés techniques mais de développer d'autres registres d'apprentissage, en lien avec des compétences dites " non techniques » (Flin, \& Maran, 2004 ; Gaba, Howard, Fish, Smith, \& Sowb, 2001). Ainsi, les objectifs pédagogiques visent le développement de compétences cognitives (raisonnement clinique, jugement clinique et prise de décision), de compétences organisationnelles (collaboration, coordination, gestion des tâches), de compétences sociales (communication, leadership/followership), et de compétences émotionnelles (gestion du stress en situation de crise). La simulation apparait comme un format privilégié pour atteindre ces objectifs.

De fait, le référentiel de formation infirmière recommande d'utiliser la simulation dans différentes unités d'enseignement, en particulier dans les unités intégratives (ministère de la Santé et des Sports, 2009). Ces dernières visent l'intégration des savoirs et la construction d'une posture professionnelle, et s'appuient sur une "pédagogie des situations " (Pastré, 2011). Les unités d'intégration sont au nombre d'une par semestre de formation (soit six semestres) et prévoient la mobilisation et l'articulation des apprentissages réalisés dans le semestre en cours et les semestres antérieurs. Les situations professionnelles ainsi travaillées peuvent être en lien avec:

- La compétence 1 : « Analyser une situation clinique et établir un diagnostic dans le domaine infirmier »;

- La compétence $4:$ «Mettre en œuvre des soins à visée diagnostique et thérapeutique »;

- La compétence $6:$ «Communiquer et conduire une relation dans un contexte de soins »;

- Ou la compétence $9:$ : Organiser et coordonner des interventions soignantes ».

L'usage de la simulation et le nombre de séances sont laissés au libre choix des équipes pédagogiques. Elle peut représenter une ou deux séances d'une demi-journée ou d'une journée par semestre et par étudiant, rarement davantage compte-tenu du coût important en temps et en organisation que le format requiert pour des promotions d'une centaine d'étudiants en moyenne, sachant que ce format exige des groupes restreints de six à dix apprenants au maximum. Ces séances peuvent être proposées en amont des stages afin de préparer les étudiants aux problématiques de soins qu'ils vont rencontrer.

Les séances de SCPE sont structurées en trois étapes qui s'enchainent: le briefing, l'exercice simulé et le débriefing. Pour le formateur qui conçoit et anime ces séances, il s'agit d'une activité complexe, comportant un degré élevé d'incertitude et des moments critiques que Pastré (1997) a identifiés :

- Lors du briefing, il est question de contrat didactique. Celui-ci implique que le sujet s'engage dans l'action comme s'il était en situation réelle, tout en acceptant les limites d'un contexte de réalisme relatif, que le formateur s'efforce de rendre le plus crédible possible. Le contrat inclut la dévolution du problème (Brousseau, 1986), puisque la responsabilité du contenu didactique produit en situation est dévolue à l'apprenant. Le briefing doit en outre informer les apprenants sur les objectifs d'apprentissage visés et leur permettre de se sentir en sécurité pour pouvoir s'impliquer dans un exercice qui les expose au regard de leurs pairs. Le formateur s'engage alors à créer les conditions favorables pour qu'un climat de travail sécure puisse se construire et se maintenir au sein du groupe tout au long de la séance (Rudolph, et al., 2007, op.cit.) ; l'enjeu est important puisqu'il s'agit d'obtenir le matériau expérientiel support d'apprentissage ;

- Au moment de l'exercice simulé, les apprenants, seuls ou à plusieurs, sont immergés dans l'environnement clinique reconstruit. Ils reçoivent la consigne de prendre en charge un 
patient présentant des caractéristiques et une problématique de soins particulières, dans un contexte qui sera précisé en amont et/ou découvert en situation. La personne soignée est simulée soit par un mannequin "haute-fidélité » piloté par un formateur (le mannequin pouvant reproduire un certain nombre de signes cliniques qui varieront selon les actions réalisées ou non), soit par un acteur qui joue le rôle du patient. Le scénario conçu par les formateurs prévoit les conditions de la confrontation des apprenants au problème qu'ils devront tenter de résoudre et les ressources qu'ils pourront rechercher (appeler un médecin par exemple). Pastré précise que l'enseignant doit veiller à gérer en temps réel le déroulement du scénario de manière à ce que l'activité visée puisse être réalisée. C'est là l'activité de guidage que nous nous proposons d'étudier ;

- Enfin, au moment du débriefing, les protagonistes sont amenés à visiter leurs processus de pensée pour expliciter ce qui les a conduits à faire telle ou telle action (ou ne pas la faire), à dire telle parole, à prendre telle décision, à avoir tel comportement. Ainsi, les représentations mentales de la situation sont évoquées, les intentions exprimées, les actions explicitées, le raisonnement clinique mis au jour, les connaissances mobilisées confrontées, les difficultés rencontrées partagées. Dans cet exercice, le formateur veille à construire un climat favorable aux interactions sociales au sein du groupe afin qu'il soit propice au raisonnement et à l'élaboration des connaissances professionnelles.

8 Le rôle du formateur est ainsi envisagé dans une fonction de facilitation des processus d'apprentissage en jeu. Le terme "facilitator», utilisé dans le monde anglophone des formations de santé, désigne le formateur se réclamant d'une approche socioconstructiviste, dans son activité globale de conduite de séances de simulation ( International Nursing Association for Clinical Simulation and Learning, 2016), à la fois lors du briefing (en facilitant l'enrôlement des apprenants dans la simulation), au cours de l'exercice simulé (en facilitant le déroulement du scénario et l'activité des apprenants) et lors du débriefing (en facilitant la prise de parole, la mise en débat et l'exploration des processus de pensée).

9 L'activité de facilitation au cours de l'exercice simulé est évoquée dans le guide de bonnes pratiques en matière de simulation (Granry, \& Moll, 2012, p. 15) en tant qu'« ajustement permanent du scénario de manière à maintenir les apprenants en situation de résolution de problème(s)». Il y est précisé que le formateur peut intervenir lui-même ou via un « facilitateur » pour aider les apprenants, en particulier pour éviter la situation d'échec et notamment le « décès » du mannequin. Mahoudeau et Berton (2013, p. 295) la présentent sous la forme d'un algorithme décisionnel permettant d'envisager les «différents chemins ", mais insistent sur le caractère imprévisible des choix des apprenants, nécessitant vigilance, réactivité et créativité, ainsi que souplesse et flexibilité de la part des formateurs.

10 Afin de distinguer facilitation et guidage, nous proposons de considérer la facilitation comme le processus global de médiation, dont le but est d'assurer le bon déroulement de la séance dans ses trois étapes. La facilitation fait référence à une posture pédagogique relative à une manière de susciter l'apprentissage dans le contexte général des pédagogies actives. Quant au guidage, il évoque plus spécifiquement l'intervention du formateur sur le couplage sujet-situation en cours d'exercice simulé. Ainsi, la facilitation inclut le guidage, mais ne se réduit pas au guidage.

11 Cette activité complexe requiert la capacité de faire face à diverses exigences. Or, elle ne semble pas faire l'objet d'un enseignement spécifique au cours des formations de formateurs à la simulation. Ces dernières consistent en des formations courtes ou des 
diplômes universitaires. Elles sont, pour la plupart, basées sur des pédagogies actives et comprennent la conception et la mise en œuvre de scénarios. Pour autant que nous en jugions d'après les contenus proposés et les témoignages de formateurs, l'enseignement relatif à la manière de guider n'y est pas explicitement formalisé. L'apprentissage de cette capacité se fait donc sur le tas, par tâtonnement, ce qui pose la question de sa transférabilité. Par ailleurs, si la conduite du débriefing a fait l'objet d'études en particulier dans la littérature anglo-saxonne (Dreifuerst, 2012; Rudolph, et al., 2007), le guidage en cours d'action est peu abordé en tant que tel dans les travaux de recherche actuels. Mieux comprendre cette activité pourrait apporter une contribution en vue de la formation des formateurs.

\section{Les recherches sur l'activité de guidage du formateur en simulation}

Les études relatives à la simulation s'inscrivent principalement dans les cadres théoriques de l'ergonomie, de la psychologie ergonomique et d'un courant qui en est issu: la didactique professionnelle. Samurçay (2005) désigne par "situation simulée» des situations qui mettent en scène un modèle d'environnement, un modèle de matériel de médiation (le simulateur), un modèle de dispositif opérationnel (l'organisation de la mise en situation des acteurs) et des scénarios (qui utilisent ou non des simulateurs physiques). La composante physique (technique ou matérielle) se réfère au domaine d'activité (ici les soins) et à la situation de référence. La composante didactique se rapporte quant à elle au modèle de compétences visées, à ses modalités de construction, ainsi qu'aux médiations nécessaires pour permettre cette construction.

13 Le simulateur est envisagé comme un instrument de formation et possède un statut d'artéfact, lequel est utilisé dans une visée de médiation entre l'apprenant et le savoir visé (Rabardel, 1995). Cependant, la médiation instrumentale ne peut être isolée de la médiation humaine réalisée par le formateur car celui-ci pilote le simulateur et anime le débriefing. Il est aujourd'hui admis que la manière dont le formateur conduit sa séance est déterminante pour augurer de la qualité et de l'efficacité pédagogique de ces formations (Boucheix, 2015 ; Rogalski, \& Samurcay, 1998; Wagemann, \& Percier, 1995). Dans le débriefing, le formateur est considéré comme un médiateur qui doit aider l'apprenant à passer du vécu au récit, et du récit à la construction de l'intrigue ou du problème, afin de conceptualiser son action, pour préparer le transfert (Pastré, 2005). Si le réalisme des simulateurs et des situations est recherché, les partisans de la didactique professionnelle considèrent qu'il est loin d'être le critère essentiel de leur efficacité dans le développement des compétences visées : «les conditions d'utilisation des simulateurs (les caractéristiques des situations didactiques qu'on construit et la manière dont elles sont menées par l'instructeur) jouent un rôle aussi - sinon plus - important que les caractéristiques du système technique lui-même » (Rogalski, \& Samurçay, 1998, op. cit., p. 337).

Envisager l'activité de guidage nécessite de la replacer dans l'ingénierie d'une séance, de sa conception à sa mise en œuvre. Lenoir et Pastré (2008) caractérisent la situation de simulation selon trois dimensions : la fidélité, la problématicité et l'interactivité :

- La fidélité vise à reconstituer un environnement le plus proche possible de l'environnement réel de travail afin de permettre la réalisation de l'activité cible. Au cours de l'exercice simulé, l'apprenant doit être en capacité de réaliser l'activité de soins dans toutes ses 
dimensions (technique, relationnelle, organisationnelle, etc.) et il doit pouvoir s'y engager et « s'y croire » (Nyssen, 2005). Pour autant, c'est davantage la fidélité à l'activité réelle qui est susceptible de générer l'immersion fictionnelle que la fidélité à l'environnement de travail (Horcik, \& Durand, 2011 ; Pastré, 2005, op. cit.) ;

- La problématicité consiste à identifier l'objectif-obstacle auquel on souhaite confronter les apprenants ; il s'agit alors de concevoir un scénario qui va permettre cette confrontation, et par conséquent, d'identifier les prérequis et les conditions nécessaires pour que les apprenants soient en mesure de gérer le problème en restant dans leur zone proximale de développement (ZPD) (Vygotski, [1934] 1997). Au cours de l'exercice, l'enseignant doit situer son intervention de guidage dans cette zone proximale pour permettre à l'apprenant de dépasser ses compétences actuelles grâce à une activité conjointe avec le médiateur ou un pair. En effet, si les étudiants infirmiers, faute de pratique suffisante en termes de savoirfaire procédural, buttent sur une difficulté technique (par exemple, savoir utiliser un défibrillateur semi-automatique), il y a risque qu'ils restent focalisés sur cet acte alors que le scénario visait d'autres objectifs (la coordination au sein de l'équipe pour la gestion de l'urgence). Dans un tel cas, le guidage par un formateur-facilitateur devient nécessaire en faisant par exemple intervenir un "collègue » qui se propose de prendre en charge la mise en place du défibrillateur, de manière à relancer l'apprenant sur la coordination des tâches ;

- Enfin, la troisième dimension est l'interaction: la situation évolue et se transforme en fonction des actions (réalisées ou non, pertinentes ou non) des apprenants. Quoi que fasse l'apprenant, le formateur doit être en mesure d'apprécier s'il faut qu'il fasse intervenir un facilitateur pour le guider. Il convient donc d'imaginer les options prévisibles, de façon à pouvoir distiller des indices adéquats, crédibles et suffisants pour orienter les apprenants, tout en laissant à ces derniers une marge d'autonomie de décision et d'action. Le formateur est ici un ingénieur pédagogique qui conçoit et gère en temps réel un environnement d'apprentissage dynamique ouvert (Rogalski, \& Samurçay, 1998, op.cit.). Il s'agit de « conception continuée dans l'usage " (Rabardel, 1995, op. cit.) puisque le formateur va composer avec le matériau pédagogique "en train de se faire" au cours de la situation simulée, matériau dont il lui faudra aussi évaluer les éléments à fort potentiel d'apprentissage pour conduire ensuite le débriefing.

Pour Simoneau et Paquette (2014, p. 16), le facilitateur guide et soutient les apprenants. Il doit adapter la situation clinique simulée " pour qu'elle réponde aux objectifs d'apprentissage, et ce, en fonction des interventions ou non-interventions du participant ». Rogalski et Samurçay ( op. cit.) ont modélisé l'activité de l'instructeur (Figure 1). Il s'agit pour lui de manipuler des variables problématiques que sont les conséquences des actions des sujets dans la situation simulée, la complexité des systèmes techniques et organisationnels, la temporalité des processus en jeu. 
Figure 1 : Schéma de la médiation sujet-situation via l'instructeur, (Samurçay \& Rogalski, 1998, p. 350).

Figure 1: Plan of the mediation subject-situation via the instructor, (Samurçay, \& Rogalski, 1998, p. 350)

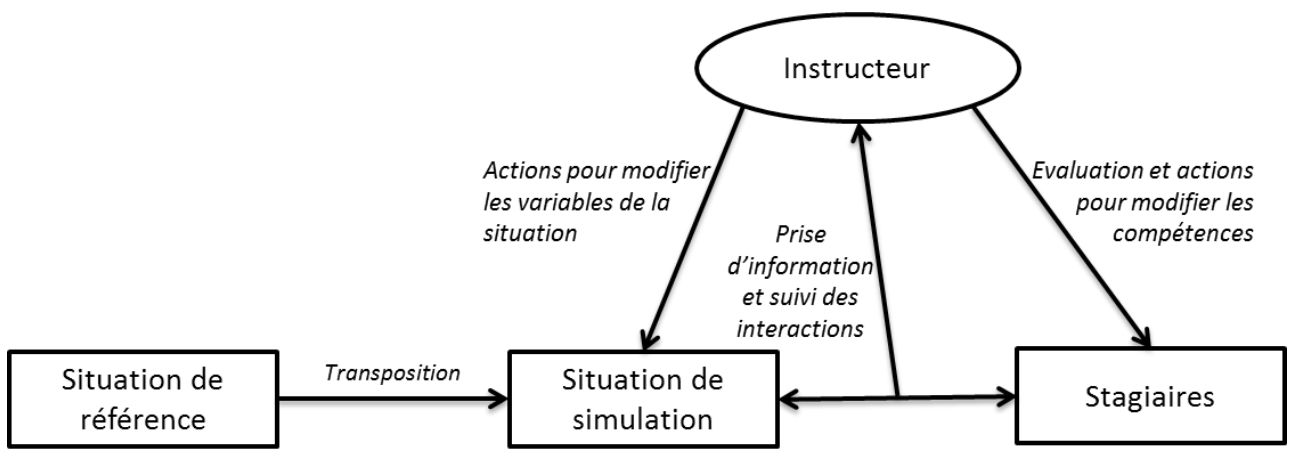

Dans cette perspective, nous pouvons considérer que, dès la conception du scénario, l'instructeur anticipe les options possibles de l'évolution de la situation et celles concernant les choix du stagiaire. Cette anticipation s'appuie sur sa connaissance des difficultés et obstacles potentiels que sont susceptibles de rencontrer les apprenants dans une situation de ce type, sur les représentations qu'il construit à propos de leurs caractéristiques (étudiants ou professionnels, novices ou professionnels confirmés, degré de familiarité supposé avec l'environnement de travail, etc.).

L'activité des formateurs est représentée comme un système de tensions entre des buts contradictoires, voire antagonistes, ce qui conduit à l'envisager sous l'angle de la régulation (Vidal-Gomel, Fauquet-Alekhine, \& Guibert, 2011, p. 136) : des régulations centrées sur la tâche et des régulations centrées sur les sujets. Les auteurs considèrent que les régulations s'opèrent entre plusieurs pôles en tension :

- Les objectifs pédagogiques, qui sont des contraintes institutionnelles mais spécifiques à l'action de formation, et que le formateur est censé atteindre ;

- Les contraintes et ressources du système : organisation du travail au sein de l'institution, équipe pédagogique formée, performante (ou pas), charge de travail des formateurs (permettant ou pas une anticipation et une préparation optimale de la séance), etc.;

- Les expériences, compétences et potentiellement le devenir du formateur: les auteurs précisent que le formateur a tendance à privilégier les champs d'intervention qu'il connait bien (relatifs à son vécu, son expérience professionnelle), ce qui peut le conduire à mettre un focus plus important sur ceux-ci ;

- Les compétences et les fonctions des apprenants : le formateur doit adapter son mode de travail pédagogique à son public et inscrire ses interventions dans la ZPD des apprenants.

18 Ce compromis est alors schématisé comme un état d'équilibre entre ces quatre pôles, lorsque ceux-ci ont un "poids» équivalent (Figure 2). Si l'un des pôles prend plus d'importance, alors le compromis se déplace vers le pôle en question. Le formateur doit alors élaborer un compromis plus acceptable au regard de ses buts et de ceux de l'institution, ou du groupe. 
Figure 2 : Quatre pôles en tension au sein desquels le formateur doit établir un compromis, (VidalGomel, Fauquet-Alekhine \& Guibert, 2011, p. 137).

Figure 2: Four poles in tension within which the trainer has to establish a compromise, (Vidal-Gomel, Fauquet-Alekhine, \& Guibert, 2011, p. 137)

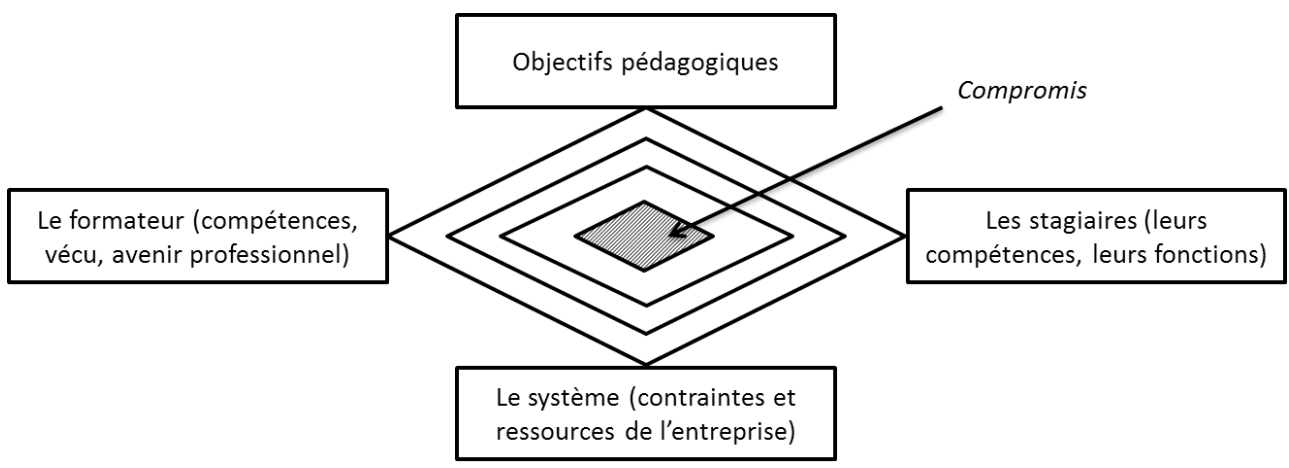

Rogalski et Samurcay (1998, op.cit.) évoquent différents travaux de recherche qui permettent d'identifier trois catégories invariantes de l'activité des formateurs: la gestion didactique de la séance (apport de savoirs, contrôle des acquis et guidage de l'activité de l'apprenant), la gestion de la simulation elle-même comme environnement dynamique ouvert (modification des paramètres de la situation) et la gestion de l'activité propre (temporalité de la séance, contrat institutionnel, etc.). Ces diverses activités varient en fonction de plusieurs variables, en lien avec le profil professionnel des stagiaires (étudiants en formation initiale ou professionnels en formation continue), la place de la séance dans le cursus de la formation (au début, à la fin), la finalité de l'aide apportée (production versus apprentissage) et le profil professionnel du formateur. Pour cette dernière, il apparait deux dimensions de la variable "profil formateur ", selon que le formateur est plutôt un professionnel de terrain ou un professionnel de la formation, et selon qu'il est novice ou expérimenté dans la conduite de simulations. Ainsi, une plus grande expérience du format est caractérisée par une plus grande autonomie laissée à l'apprenant (activité de guidage plus faible, y compris en laissant faire l'erreur). À l'inverse, les formateurs novices ont tendance à intervenir plus tôt sur l'activité des apprenants, de manière à éviter de se retrouver eux-mêmes dans une situation imprévue qui les mettrait en difficulté dans le déroulement de la situation. Autrement dit, plus l'incertitude augmente pour le formateur, plus celui-ci est interventionniste (Wagemann, \& Percier, 1995). De même, plus le formateur est expérimenté, plus il pronostique précisément les difficultés des apprenants, et donc plus il ajuste son mode d'intervention. Les formateurs "issus du rang » ont davantage de facilité à diagnostiquer les difficultés potentielles, mais pour autant, certains d'entre eux, conditionnés par leur expertise métier, ont tendance à apporter une aide orientée vers la réussite de l'action au détriment de sa compréhension. Wagemann et Percier (op. cit.) montrent que l'activité des instructeurs comporte deux composantes principales : une composante technique et une composante pédagogique, mais que, compte-tenu des objectifs visés et des contraintes temporelles et organisationnelles, leur logique d'expert-métier prend le pas sur la logique pédagogique. Ainsi, plus le système "situation simulée » est ouvert, plus l'incertitude est forte pour le formateur, et plus les stratégies de guidage sont des stratégies directes et de type contrôle ou correction. formateurs. La première est liée à l'activité de production : le formateur est centré sur la 
situation et ses interventions visent à la conduire en référence au modèle du réel. Il va veiller à respecter au plus près le réalisme de la situation de travail, guider l'apprenant afin que celui-ci évite de faire des erreurs et se focaliser sur le respect des procédures prescrites. Plus l'environnement est reproduit de façon réaliste, plus cette logique est privilégiée. La seconde logique est plutôt centrée sur l'apprenant: dans ce cas, le formateur considère l'activité de l'apprenant comme une activité d'apprentissage et la question de la conformité ou non-conformité aux normes ou aux bonnes pratiques devient un matériau d'analyse pour le débriefing.

Quant à la nature des interventions de guidage, Vidal-Gomel, Boccara, Rogalski, et Delhomme (2008), étudiant les formateurs en conduite automobile, envisagent le guidage comme une activité de tutelle, au sens où elle a été conceptualisée par Bruner (1983), c'est-à-dire "les moyens grâce auxquels [...] un spécialiste vient en aide à quelqu'un qui [...] est moins spécialiste que lui ». Les auteurs reprennent trois des six fonctions de la tutelle: la signalisation des caractéristiques déterminantes de la situation, la réduction des degrés de liberté et la démonstration. La signalisation correspond à l'activité du formateur destinée à signifier à l'apprenant les caractéristiques de la tâche qui sont pertinentes pour son exécution: par exemple attirer l'attention sur un élément important de la situation, corriger ou expliquer. La réduction des degrés de liberté consiste à simplifier la tâche par réduction du nombre des actes requis pour parvenir à la solution : autrement dit, il découpe la tâche en sous-buts que l'apprenant pourra plus facilement gérer. La démonstration vise à montrer à l'apprenant comment faire afin qu'il soit en mesure d'exécuter lui-même la tâche. Les auteurs identifient deux types de guidage chez ces formateurs. Le premier est focalisé sur la signalisation des caractéristiques pertinentes de la tâche. Le second type de guidage est plus diversifié avec un nombre d'interventions moins élevé en cours d'action.

Dans une perspective proche, Cardin (2016) étudie l'activité d'instructeurs sapeurspompiers dans le contexte de la formation en simulation des chefs d'agrès. Il reprend aussi trois fonctions de la tutelle, selon Bruner (1983): la signalisation des caractéristiques pertinentes, la réduction des degrés de liberté et le maintien de l'orientation. Cette dernière fonction vise à éviter que l'apprenant ne s'écarte du but assigné : il permet de le recentrer sur la tâche précise. Par exemple, le formateur fait intervenir un témoin ou un tiers (ou joue lui-même ce rôle) pour donner une information ou une consigne de façon à guider l'apprenant et le rediriger lorsqu'il s'égare. Cardin met en évidence que l'activité du formateur s'oriente préférentiellement vers le pôle productif, selon un mode de régulation direct : il gère les variables de la situation simulée dans une perspective de réalisme optimal. En revanche, le pôle didactique est sousinvesti: il y a peu de régulation indirecte, sauf lorsque le formateur estime que l'apprenant est hors-sujet ou qu'il est dépassé par la complexité du problème à gérer. Ainsi, la gestion des variables de la situation simulée est privilégiée au détriment d'une gestion didactique, ce qui rejoint les résultats mis en évidence dans les recherches antérieures.

Dans la littérature relative à la simulation en formation infirmière, principalement anglosaxonne, des standards de bonnes pratiques ont été publiés par l'International Nursing Association for Clinical Simulation and Learning (INACSL, 2016) en lien avec l'activité de guidage du formateur, lequel apparait davantage centré sur l'apprenant. Ces normes recommandent notamment que le guidage soit "adapté au niveau d'apprentissage, à l'expérience et aux compétences des apprenants et qu'il comporte la délivrance d'indices 
prédéterminés et/ou non planifiés dans le but d'aider les personnes à atteindre les objectifs ${ }^{1}$. Dans le milieu francophone, Horcik (2014) a étudié les pratiques de formateurs dans des séances de simulation clinique destinés à des infirmiers suivant un cursus de formation spécialisée en anesthésie. L'auteure met en évidence la difficulté pour les formateurs à capter l'activité durant son déroulement, compte tenu de sa rapidité (ce sont des situations d'urgence où des actions multiples sont conduites simultanément par plusieurs acteurs).

Au terme de cette revue de littérature, nous pouvons dire que l'activité de guidage est vue comme un processus de régulation et qu'elle révèle une mise en tension au sein d'un système de contraintes. Si la nature de cette activité est de l'ordre de la tutelle, elle répond à plusieurs objectifs que le formateur semble devoir gérer en fonction des priorités qu'il identifie. Il existe donc une composante cognitive en parallèle de la composante médiative. L'étude de ces deux composantes permet d'identifier assez précisément les logiques d'action des sujets, selon Robert et Rogalski (2002). La composante cognitive traduit les évaluations et les décisions du formateur à partir de ce qu'il observe de la situation simulée. La composante médiative correspond à son action sur la situation et les sujets, et à ses modes d'interaction (directe ou indirecte) : accompagnement des apprenants, gestion de l'artéfact, nature et tonalité du discours, improvisations, délivrance d'indices, etc.

Mettre en visibilité cette activité revêt selon nous un enjeu important : celui d'identifier ses déterminants et ses contours, afin d'optimiser la construction de la compétence de guidage dans les programmes de formation de formateurs. D'un point de vue opérationnel, trois raisons justifient cette focalisation. La première est qu'un guidage efficace permet la production d'un matériau didactique qui sera en cohérence avec les objectifs d'apprentissage espérés. S'il n'est pas efficace, la situation peut présenter une évolution différente de celle souhaitée, faisant émerger d'autres problématiques (par exemple un conflit interprofessionnel alors que ce n'est pas l'objectif ou, à l'extrême, conduisant au décès du "patient»). Ces évolutions imprévues - bien que parfois intéressantes - ne répondraient pas toujours aux objectifs visés par l'équipe pédagogique. La deuxième raison est qu'un guidage défaillant risquerait de confronter l'apprenant à l'échec sous le regard de ses pairs, ce qui pourrait compromettre ses apprentissages futurs et son sentiment d'efficacité. Troisièmement, un guidage qui ne rendrait pas suffisamment crédible l'évolution clinique de la situation risquerait de nuire à l'adhésion des apprenants à l'expérience simulée et à leur immersion fictionnelle dans l'exercice, ce qui mettrait en péril le matériau didactique destiné à l'analyse réflexive. Aussi, comprendre les mécanismes cognitifs qui sous-tendent ces interventions et la manière dont elles sont mises en œuvre nous parait un point focal à explorer et constitue à notre avis un champ d'exploration possible pour prolonger les travaux de recherche actuels.

\section{3. Étude des modalités de guidage au cours de l'exercice simulé}

\subsection{Contexte de la recherche et échantillon}

Nous nous proposons d'étudier ces modalités de guidage à partir de l'observation des pratiques des formateurs, puis de leur discours sur cette activité, afin d'identifier les logiques d'action qui les sous-tendent. En complément nous utilisons, à titre 
d'illustrations, du matériau empirique récolté en amont au cours d'une étude exploratoire auprès de cinq formateurs experts en simulation clinique.

Notre panel est constitué de seize formateurs en soins infirmiers, pour la plupart cadres de santé. Seuls deux d'entre eux sont des infirmiers ayant le projet d'entrer en formation cadre $^{2}$. Ils exercent à temps plein leur activité pédagogique dans un Ifsi depuis sept ans en moyenne (de 1 à 17 ans). Ils rencontrent les étudiants tout au long de l'année dans tout type de formats pédagogiques (cours, séances de travaux dirigés ou de travaux pratiques, etc.). La simulation représente une part assez modeste de leur activité globale (6 à 30 séances par an) sauf pour deux d'entre eux dont c'est l'activité principale. Au moment de la séance observée, leur expérience de la SCPE est de quatre ans en moyenne (de 1 à 16 ans). Tous ont choisi de s'engager dans cette activité et ont suivi une formation spécifique (formation courte dans un centre de simulation); trois d'entre eux sont titulaires d'un diplôme universitaire de formateur en simulation. Treize formateurs sur seize ont exercé auparavant comme infirmier ou comme cadre dans un service d'urgence ou de réanimation, ce qui n'est pas surprenant compte tenu que la simulation en santé s'est d'abord développée dans le contexte de l'enseignement des soins d'urgence.

Les trois instituts enquêtés disposent d'un laboratoire de pratiques simulées in situ et/ou ont accès à un centre de simulation. Dans chaque Ifsi, l'activité simulation est structurée de la même manière: un groupe de pilotage, constitué des formateurs les plus expérimentés en SCPE, est chargé de concevoir des scénarios, d'organiser l'activité sur un plan logistique et de superviser les collègues novices dans la conception et la mise en œuvre des séances. Neuf formateurs du panel sont membres de ces groupes de pilotage et ont été initiateurs de l'activité au sein de leur Ifsi quatre ou cinq ans plus tôt. Les sept autres se sont engagés plus récemment. La plupart du temps, les séances de simulation sont conduites simultanément par deux formateurs, compte-tenu de la pluralité des tâches à gérer: piloter le mannequin, observer et analyser la scène, intervenir dans la situation, parfois même jouer le rôle du patient. Les formateurs évoquent tous la plusvalue pédagogique de ces séances qui permettent, selon eux, de faire le lien entre la théorie et la pratique de terrain. Le retour généralement enthousiaste des étudiants les conforte en ce sens.

Les séances observées s'adressaient à des étudiants infirmiers de la première à la troisième année de formation. Elles se situaient dans le cadre des unités d'enseignement «soins d'urgence» ou "soins relationnels» ou encore dans le cadre des unités intégratives visant l'acquisition de compétences professionnelles, comme nous l'avons évoqué supra. Les étudiants avaient bénéficié en amont de cours et de travaux pratiques relatifs aux prérequis nécessaires pour aborder la prise en charge en question. Le degré de complexité des scénarios variait en fonction du niveau d'études : les scénarios relatifs à la gestion de situations d'urgence ou de situations relationnelles complexes, étaient destinés à des étudiants de deuxième ou de troisième année. Chaque séance comportait deux à quatre scénarios et se déroulait sur une demi-journée ou une journée. Le simulateur était soit un mannequin, soit un comédien ou un formateur jouant le rôle du patient. Les étudiants (par groupe de six à dix personnes) avaient revêtu leur tenue professionnelle; certains, à l'issue du briefing, s'étaient portés volontaires pour la situation. Les protagonistes étaient alors invités à entrer dans la chambre pour prendre en charge le patient, seuls, à deux ou à trois, selon le type de scénario. Lors du briefing, ils recevaient des informations relatives à l'état clinique du malade, au contexte d'hospitalisation et à la nature de la tâche à réaliser (par exemple : conduire un entretien 
d'accueil, procéder à l'observation clinique de la personne, ou encore être appelé à son chevet par l'aide-soignant car « le patient ne va pas bien»).

Dès que les étudiants entraient en action, l'un des formateurs en régie pilotait le mannequin (voix du patient et gestion des paramètres cliniques) tandis que l'autre observait la situation afin d'en analyser l'évolution et décidait des interventions de guidage à réaliser. Il prenait généralement le rôle du médecin appelé en renfort ou celui d'un autre professionnel. C'est souvent ce formateur qui conduisait ensuite le débriefing. Parfois les enseignants étaient tous deux en régie et se concertaient sur les choix de guidage à opérer. D'autres fois, le formateur-débriefeur observait la scène retransmise sur écran vidéo, avec le groupe d'étudiants observateurs restés en salle de débriefing. La situation durait généralement une dizaine de minutes.

31 Nous avons aussi regardé les documents dont se servent les formateurs comme supports de leur action. Ces derniers utilisent un conducteur pédagogique, comparable d'un Ifsi à l'autre, comprenant différents éléments. D’abord la thématique générale, par exemple : « Prise en charge d'un patient dans un contexte infectieux, avec dégradation de l'état clinique » et la contextualisation de l'histoire clinique du patient, qui sera présentée aux étudiants lors du briefing. Figurent aussi l'objectif principal du scénario («Permettre à l'étudiant de reconnaitre les éléments de gravité d'une situation d'urgence et d'apporter une réponse adaptée ») et les objectifs d'apprentissage «secondaires" déclinés dans les domaines d'apprentissage cognitif, technique et comportemental. Les consignes de préparation de la salle sont indiquées et les paramètres cliniques du mannequin en situation initiale et en situation "dégradée » sont précisés. Concernant le guidage en situation, nous retrouvons une "fiche rôle patient »: "à l'arrivée des étudiants, vous vous plaignez d'avoir froid, de frissons, de fatigue. En fonction des questions qu'ils vous posent, vous dites "ça dure depuis une demi-heure" [...]. Vous demandez une couverture. Vous êtes angoissé ». Nous trouvons aussi une "fiche rôle infirmière du service »: "en réponse à l'appel des étudiants, vous ne pouvez pas venir tout de suite. Vous demandez les constantes (si non dites), les signes cliniques (si non dits), vous dites "j'arrive dès que possible" ", sans qu'il y ait davantage de précision. Des consignes pour le rôle du médecin sont retrouvées dans d'autres documents mais sont généralement constituées de quelques phrases-type assez succinctes tel le modèle suivant : "si les étudiants disent ceci, dire cela », «s'ils ne font pas ceci dans les 5 minutes, faire cela ». Les formateurs ont le document à portée de main mais ils ne le regardent que peu en cours d'action, celle-ci requérant une réponse immédiate - ce qui suppose qu'ils se soient imprégnés du scénario en amont.

32 Afin de permettre au lecteur de se représenter l'intervention de guidage, nous évoquerons ici l'un des scénarios, assez emblématique, mettant en scène un patientmannequin dont l'état clinique va se dégrader. Deux étudiants infirmiers sont appelés à son chevet, en l'absence de l'infirmière occupée ailleurs. L'objectif d'apprentissage est en lien avec la construction de compétences cognitives, gestuelles et sociales dans le contexte des soins d'urgence : raisonnement clinique à partir des paramètres vitaux mesurés et des dires du "patient ", identification d'un diagnostic de situation clinique (reconnaitre l'évolution péjorative vers un état de choc septique), prise de décision concertée et mise en œuvre coordonnée des actions requises (appel téléphonique au médecin, mise en œuvre de mesures conservatoires).

Dans ce scénario, le formateur s'attend à intervenir à plusieurs niveaux :

- Faire varier les paramètres cliniques du mannequin en fonction des actions attendues, réalisées (ou pas) par les étudiants (par exemple, s'ils surélèvent ou pas les jambes du 
patient, s'ils lui mettent ou pas un masque à oxygène, s'ils mesurent des paramètres (et lesquels), etc.) ; dans ce cas, le formateur augmente ou fait baisser la pression artérielle, augmente ou fait baisser la saturation en oxygène, etc., en fonction de l'efficacité clinique prévisible pour chaque action réalisée ou non ;

- Répondre à l'appel téléphonique en jouant le rôle du médecin ou de l'infirmière; le formateur ajuste son intervention à la demande de l'étudiant et distille des indices en fonction de ce qui lui est dit ou pas: selon la précision et la pertinence des données transmises par les étudiants, le « médecin » reconnaitra ou pas l'urgence à se déplacer ;

- Décider d'entrer lui-même dans la situation ou faire intervenir un compère s'il le juge nécessaire pour aider à débloquer une situation qui s'enlise, ou complexifier une situation trop facilement gérée; dans ce cas, il évalue les besoins et décide de la nature de l'intervention à opérer (soutien sur une tâche, perturbation).

En fonction des exigences - toujours singulières - requises par la situation et les apprenants, le formateur dispose d'une marge d'autonomie par rapport au référentiel planifié. Il régule ses interventions à partir des informations qu'il relève et interprète. Il choisit alors d'intervenir soit sur les variables de la situation, soit sur l'activité des sujets, généralement sur les deux. Même si le formateur n'intervient que de façon ponctuelle au cours de la situation simulée, il en est un acteur à part entière, de façon directe et/ou indirecte. À ce titre, il devient lui-même une variable de la situation problème, dans la mesure où il va aider à la résolution du problème (faciliter l'action) ou au contraire constituer une partie du problème (ajouter un aléa), en fonction du niveau de difficulté souhaité.

\subsection{Méthodologie}

Le matériau empirique analysé a été obtenu au cours d'observations de séances suivies d'entretiens d'autoconfrontation. L'activité du formateur au moment de l'exercice a été enregistrée et a fait l'objet de commentaires de la part du chercheur. Lors de l'entretien d'autoconfrontation, nous avons demandé à chacun d'expliciter quelles avaient été ses priorités et ses intentions, ce qui l'avait conduit à opérer tel ou tel choix, et comment il les avait traduits en termes d'action, à différents moments "critiques", autrement dit aux moments nécessitant une intervention directe ou indirecte de sa part. Bien que nous n'ayons observé qu'une seule séance par formateur, l'entretien d'autoconfrontation a permis de repérer si le comportement explicité était revendiqué comme un principe d'action stabilisé ou s'il était plutôt réactionnel au contexte singulier.

Les données ont été analysées selon une méthode de catégorisation conceptualisante (Paillé, \& Mucchielli, 2012). Cette méthode, reprenant les principes de la théorisation ancrée (Glaser \& Strauss, 1967), donne à voir la réalité du terrain, le motif d'une interaction et permet de croiser les pratiques observées et les discours dans une recherche de sens; la catégorie est vue comme outil de conceptualisation. Nous avons procédé par induction à partir des données recueillies pour construire des catégories spécifiant différentes modalités de guidage en fonction de leur cible, de leur but ou de leur logique. Progressivement, selon un processus itératif, sept catégories ont émergé, décrivant la nature de ces modalités. Certaines se sont naturellement rapprochées des concepts mis en évidence dans les recherches présentées supra. Chaque catégorie a été caractérisée par ses propriétés et ses conditions d'existence, et a été stabilisée dans la mesure où nous retrouvions chez plusieurs formateurs des indicateurs la décrivant. Par 
exemple, la catégorie "guidage instrumental » décrit les interventions à destination de l'artéfact. Elle est donc soumise à la présence d'un instrument nécessitant une régulation directe de la part du formateur, plutôt de nature «technique»: ici le mannequin à « animer » et ses paramètres à modifier. Cependant le fait que, dans certaines séances (soins relationnels), le patient soit une personne physique justifie un guidage indirect (en amont de l'exercice pour lui donner des consignes de jeu ou en aval pour lui demander de réajuster son jeu). Nous considérons cependant ce guidage comme instrumental car sa cible est l'artéfact (qu'il soit humain ou synthétique), ainsi que nous l'identifions par exemple chez Clément ${ }^{3}$ vis-à-vis des comédiens-patients, à propos d'une situation de relation avec un patient agressif :

CLÉMENT : Je les ai aussi briefés sur le fait qu'on pouvait soit augmenter, soit temporiser l'action, au besoin et à la vue de la simulation. [...] Je voulais vraiment interagir sur le curseur. De manière à ne pas mettre les étudiants en difficulté. Voilà. Et ça, les comédiens l'ont très bien compris et ont même été plutôt satisfaits qu'il y ait justement ce fameux curseur modulable... d'être entre le... suffisamment angoissé, suffisamment débordant d'agressivité pour pouvoir être géré, mais pas trop. [...] Donc on avait convenu d'un petit signe, voilà.

\subsection{Résultats : sept modalités de guidage}

Tout d'abord - et c'est révélateur de son caractère implicite - l'activité de guidage en cours d'exercice n'est pas évoquée spontanément par les formateurs lors des entretiens. Ils priorisent d'abord l'enrôlement des étudiants dans l'exercice (car celui-ci peut susciter des résistances), ils s'attachent ensuite à la maitrise de la dimension technique (savoir faire fonctionner le mannequin via la console informatique, la vidéo, le son et gérer les aléas techniques) et enfin à la conduite du débriefing (lequel étant considéré par tous comme un exercice difficile). L'activité de guidage est donc une activité construite empiriquement, pas forcément théorisée, ce qui nous conforte dans la nécessité de l'explorer.

Les sept modalités de guidage identifiées sont de nature instrumentale, étayante, didactique, prospective, de commodité, productive et normative. Le tableau 1 ci-dessous présente succinctement leur intitulé, leur cible et le but visé par le formateur. Nous les détaillerons ensuite plus précisément.

Tableau 1 : Sept modalités de guidage, leur cible et le but poursuivi par le formateur. Table 1: Seven modalities of guidance, their target and the purpose pursued by the trainer

\begin{tabular}{|l|l|l|}
\hline \multicolumn{1}{|c|}{ Modalité de guidage } & \multicolumn{1}{|c|}{ Cible } & \multicolumn{1}{c|}{ But poursuivi par le formateur } \\
\hline Guidage instrumental & Artéfact & Piloter le mannequin ou diriger le patient simulé \\
\hline Guidage étayant & Apprenant et son activité & Maintenir l'apprenant dans sa ZPD \\
\hline Guidage didactique & Situation didactique & Viser la confrontation à l'objectif-obstacle \\
\hline Guidage prospectif & Débriefing & Obtenir le matériau didactique pour le débriefing \\
\hline Guidage de commodité & Formateur & Rester dans sa zone de confort \\
\hline Guidage productif & Réalisme clinique & Rechercher la meilleure fidélité à la situation de référence \\
\hline Guidage normatif & Référentiel & Respecter le programme décidé en équipe \\
\hline
\end{tabular}


39 Précisons toutefois que ces modalités ne sont pas exclusives les unes des autres. Elles sont souvent complémentaires, parfois simultanées, quelquefois consécutives. Nous les retrouvons chez la plupart des formateurs mais à des degrés divers, ce qui oriente vers une certaine différenciation des profils des formateurs, en fonction de priorités dans l'usage qu'ils font de la simulation. Nous voyons aussi d'emblée que certains buts peuvent être simultanément pluriels ; ainsi, comme nous le percevons dans le discours de Clément (cité supra), le but de la régulation qu'il exerce vis-à-vis du comédien se traduit à la fois dans un guidage instrumental (le diriger de façon à ce qu'il interagisse comme souhaité avec l'étudiant) et, indirectement, dans un guidage étayant (garder l'étudiant dans sa ZPD).

Nous présentons ces modalités depuis les plus communes à tous les formateurs jusqu'à celles qui paraissent plus discriminantes. Pour chacune, nous évoquons sa nature, sa cible et l'illustrons avec des extraits de discours expliquant les buts que les formateurs poursuivent.

\subsubsection{Un guidage « instrumental » à destination de l'artéfact}

41 Le guidage instrumental est ciblé sur le simulateur et vise à assurer le déroulement du scénario dans une logique plutôt techniciste. Il est toujours présent et partiellement planifié en amont lors de la conception, ainsi que nous l'avons évoqué plus haut. Pour le mannequin, le guidage consiste généralement dans un premier temps à opérer le passage d'une situation dite "de base » à une situation dite "dégradée » (généralement un état clinique défaillant) constituant le problème à gérer. Dans un second temps, l'intervention vise à s'ajuster aux actions mises en œuvre (ou pas), afin de faire évoluer la situation sur un plan clinique, généralement vers l'amélioration et la stabilisation relative de l'état clinique du patient. Le guidage instrumental est ainsi simplifié au maximum, le nombre de paramètres à faire varier étant assez limité dans les scénarios observés (tension artérielle, pouls, saturation en oxygène, ouverture/fermeture des yeux). Les formateurs modulent ces variations via la console de commande du mannequin ou le micro-voix du patient, en fonction des objectifs opérationnels à atteindre, des connaissances cliniques qu'ils possèdent et de leur appréciation de l'évolution clinique potentielle. Pour cette dernière, certains estiment cependant faire varier le curseur temporel si la situation l'exige (par exemple retarder une chute de la tension artérielle pour donner le temps aux étudiants d'agir), ce qui signifie qu'un guidage étayant est opéré simultanément.

Nous observons généralement que plus le formateur est expérimenté, plus la palette des possibles est envisagée et plus la souplesse est grande en termes d'ajustement; cela traduit une certaine maitrise technique et une capacité de gestion de l'incertitude. Alors qu'un novice va veiller à suivre le conducteur prévu avec une ou deux variantes possibles, le formateur plus expérimenté dispose de davantage de stratégies et d'aisance pour faire progresser la situation vers l'évolution souhaitée en prenant en compte plusieurs cibles à la fois. Ainsi, le guidage de William est orienté à la fois sur l'artéfact et sur le réalisme clinique :

WILLIAM : Pendant la phase de l'élaboration, on envisage tous les possibles en fait, pour que si le groupe emprunte telle ou telle voie, on puisse proposer les modifications sur le mannequin [...] S'il fait ça, qu'est-ce que je fais? Pour modifier les paramètres du mannequin, pour que ce soit toujours une histoire crédible.

En revanche, lorsque le patient simulé est joué par un comédien, l'intervention directe en cours d'exercice est moindre, voire absente. Le guidage est alors dévolu à l'acteur-patient, 
puisque c'est ce dernier qui va devoir ajuster ses réactions et ses paroles pour orienter l'étudiant vers l'obstacle, puis vers la résolution du problème. Dans ce cas, l'intervention du formateur vis-à-vis de l'apprenant est indirecte. Parfois, comme c'est le cas pour Clément, il est convenu qu'il pourra faire un signe au comédien depuis la porte de la chambre pour lui signifier de monter ou baisser le niveau de difficulté, ce que fera ce formateur à deux reprises.

Mais il arrive parfois que l'acteur ne soit pas en mesure de moduler ce curseur. Katia évoque une perte de contrôle sur la situation alors que le mannequin ne lui pose pas ce genre de problème puisque c'est elle qui le dirige. Lors de la séance qu'elle anime, l'objectif est de confronter deux étudiants infirmiers de première année à l'accueil d'une patiente âgée à l'hôpital, dans un contexte préopératoire générant chez elle de l'anxiété. Les consignes données sont celles-ci : "Vous recevez Madame Martin, entrant ce jour en chirurgie pour une pose de prothèse de hanche. Vous réalisez l'entretien d'accueil ». L'objectif d'apprentissage vise la complétude du recueil de données et l'identification du problème d'anxiété et de ses causes. Dans le scénario présent, la comédienne montre peu d'anxiété et est assez passive, laissant l'initiative de l'échange aux étudiants. De ce fait, l'objectifobstacle est peu atteignable, et les étudiants restent dans leur zone de confort. Le discours de Katia montre qu'elle attend de la comédienne une bonne connaissance de son rôle, puisque la formatrice ne peut pas se substituer à elle, ni la guider en direct, ne disposant pas d'un système de micro-oreillette.

KATIA : Il se trouve que la prestation de l'actrice était pas très satisfaisante en fait. [...] Elle les a pas beaucoup déstabilisés. Et j'ai vu la différence avec hier, où c'était une autre comédienne, et effectivement, c'était plus en finesse. [...] c'est-à-dire si au bout de tant de temps, si les étudiants n'ont pas abordé ça, ben finalement l'actrice va peut-être dire une phrase ou apporter quelque chose pour amener les étudiants à. Le script est assez précis en fait. Donc je pense qu'elle s'en est pas suffisamment saisie.

Dans cet extrait, nous repérons l'algorithme décisionnel dévolu au comédien. Nous observons que Katia se laisse diriger par ce que l'actrice va produire ou non; d'ailleurs, elle ne lui a pas donné de consignes de réajustement entre les deux scénarios. Elle ressent que cela pose un problème mais n'identifie pas sa responsabilité dans l'ajustement de l'acteur aux capacités de l'apprenant et à l'objectif visé. L'enjeu de la formation des acteurs, briefés en amont par Irène, la référente du groupe de pilotage, n'est pas évoqué. Cette dernière relate ce qu'elle attend des comédiens :

IRENE: Alors de ne pas mettre trop en difficulté les étudiants, parce que quelquefois c'est facile, hein. Quand ils sont en difficulté, d'essayer de repérer quand ils sont en difficulté, et de les amener sur des choses un peu plus faciles. D'y aller crescendo. Le premier facile, le deuxième un peu moins et le troisième difficile.

Dans ce même scénario, mais dirigé par leur collègue Luc avec une autre comédienne, la situation évolue différemment. L'actrice, grâce à sa capacité de moduler son jeu, amène les étudiantes à prendre en compte son anxiété et à ajuster leur mode relationnel. Cela suscitera des questionnements constructifs au moment du débriefing (comment faire exprimer cette anxiété, comment se comporter avec un patient anxieux). Mais cette situation est aléatoire: Luc aurait tout aussi bien pu ne pas obtenir ce matériau didactique. Il accepte cette incertitude et la considère comme un paramètre inhérent à la simulation. Dans cet extrait de l'entretien d'autoconfrontation, il évoque ses attentes visà-vis des comédiens : 
LUC : Qu'ils soient... euh... qu'ils soient ni trop, ni pas assez facilitants. C'est-à-dire qu'il faut laisser les choses évoluer... faut pas, tu vois, faut pas qu'ils aillent trop, trop vite. Si tu donnes trop d'informations avant que... avant que les étudiants viennent les chercher, ben tu casses un peu le truc, quoi. Ils ont tout, ils ont rien à aller chercher. [...]. C'est pas facile le rôle de l'acteur, hein, c'est un rôle difficile. Ça leur demande de comprendre... de bien avoir perçu ce qu'on veut. De... de savoir mettre un petit peu les étudiants sous tension, mais pas trop non plus, tu vois, juste ce qu'il faut, donc c'est pas évident de doser ça, quoi. Mais, non, là on avait une actrice qui était bien, quoi.

CHERCHEUR : C'est un peu la loterie?

LUC : Tu fais avec. Tu fais avec. (Rires)... Tu rattrapes comme tu peux, tu vois... de toute façon, il y a quelque chose qui a été fait, il y a eu une action, donc euh... La seule chose, c'est ce que tu as prévu... tu peux pas... tu pourras peut-être pas balayer ce que tu as prévu dans tes objectifs, comme tu l'avais prévu. Mais de toute façon, il y a quelque chose qui s'est joué, donc... de toute façon, tu as matière.

Nous constatons que l'attente de Luc vis-à-vis du comédien est dirigée vers la modulation de son registre d'intervention, davantage que vers la conformité au scénario initial. Une grande latitude est ainsi laissée au comédien que Luc, formateur expérimenté en simulation, dirige peu. Pour lui, peu importe la tournure que prendra la situation, il estime qu'il pourra travailler sur ce qui aura été produit et le relier à des objectifs plus généraux. L'objectif d'apprentissage prévu initialement passe au second plan car ce qui prime pour lui est la gestion d'un problème, quel qu'il soit. Ce que vise Luc, c'est avant tout à amener les étudiants à s'interroger sur leurs pratiques et sur les problématiques de soins, pour qu'ils deviennent des praticiens réflexifs.

Dans l'Ifsi 3, les formateurs ont choisi de jouer eux-mêmes le rôle du patient. Ainsi, disent-ils, le problème du guidage vis-à-vis de l'acteur est résolu car le formateur, en tant que professionnel de santé, connait bien les manifestations de la maladie et de l'anxiété : son expérience de soignant lui permet de faire appel à ses propres souvenirs pour rendre crédibles les attitudes et réactions du patient. Ce que mettent en avant ces formateurs pour justifier leur choix, c'est qu'ils savent où placer le curseur pour que les étudiants soient en capacité de gérer le problème, en fonction des buts poursuivis et des potentialités de chacun.

\subsubsection{Un guidage « étayant » centré sur l'apprenant}

49 Le guidage « étayant » a pour cible l'apprenant dans son interaction avec la situation et le "patient ». C'est pourquoi nous voyons d'ores et déjà, avec tous les formateurs évoqués plus haut, que ces deux premières modalités sont étroitement liées, la première étant le moyen d'agir sur la seconde. En effet, il s'agit de maintenir l'apprenant dans sa ZPD afin qu'il soit en capacité de réaliser l'activité, que ce soit en agissant directement sur le mannequin, ou indirectement en dirigeant la médiation du comédien dans une logique pédagogique. Pour la plupart des formateurs, l'exercice est guidé dans une posture de bienveillance, veillant à ne jamais laisser un étudiant dans une difficulté insurmontable :

MARC : Et j'étais aussi sensible à... au vécu de l'étudiante. Pour être toujours prêt à intervenir au cas où je la sente vraiment trop mal et où il faut arrêter, quoi. Parce que il faut pas nuire à l'étudiant. On peut le mettre dans l'inconfort, ce qui est souvent le cas, mais faut être clair et vigilant pour que ça devienne pas... enfin, pour que ça se transforme pas en souffrance.

Lorsque la situation s'enlise, les formateurs n'ont parfois d'autre choix que de «stopper " la situation ou d'entrer eux-mêmes dans la scène en tant que « soignant ». 
JOSIE : C'est un peu au feeling, j'ai envie de dire. Parce qu'on se dit « qu'est-ce qu'on fait? On arrête ? On laisse ? ». [...] Il faut qu'ils se débrouillent un peu, il faut qu'ils avancent un peu. Donc on les laisse se dépatouiller. Par contre, voilà, enfin... comme la situation où on s'est dit « on arrête ou pas? » et comme l'autre étudiante elle a su gérer, ben on les a laissés faire. Pour pas justement qu'ils arrêtent sur une difficulté... ça peut-être je pense c'est important. Et du coup, c'est ça, on se dit " voyons ce qui se passe, s'ils arrivent à débloquer... ». Comme ça, on n'arrête pas sur quelque chose qui est pas satisfaisant pour la personne. Voilà, on se laisse du temps pour voir qu'est-ce qui se passe. Si ça arrive à débloquer, très bien, on laisse encore une ou deux minutes.

51 Leurs représentations des potentialités des étudiants leur permettent d'ajuster leur mode d'intervention en fonction de ce qu'ils estiment pouvoir attendre de chacun. L'intention première est d'éviter de mettre l'étudiant en échec en jouant notamment sur le curseur temporel (retarder l'évolution clinique quitte à en sacrifier le réalisme) :

SYLVIE : Quand on connait les étudiants, leur faire quelque chose d'un peu sur mesure... Par exemple, je sais que j'ai une étudiante qui a peu de confiance en elle, ben je vais commencer assez doucement, quand je vais voir qu'elle a posé ses marques, après je vais faire un peu évoluer le scénario,... et puis bon, il y a des étudiants qui ont besoin de beaucoup de challenge, alors ceux-là ben ça va aller un peu plus vite... c'est ce qui fait que ça doit être une approche qui doit être très dynamique.

Entre Josie la novice et Sylvie l'experte, nous voyons que les différentes options ont été plus ou moins réfléchies en amont, ce qui amène Sylvie à une intervention plus ajustée et moins aléatoire par rapport à Josie qui n'a pas envisagé d'autre choix que de stopper la scène si les étudiants ne parviennent pas à dépasser l'obstacle. Nous retrouvons cette difficulté à anticiper chez tous les novices, ainsi que chez certains formateurs plus expérimentés lorsqu'ils sont dépendants de la médiation d'un comédien.

La plupart des formateurs ont le souci de ne pas mettre l'étudiant en échec. En référence aux fonctions de tutelle définies par Bruner (1983, op. cit.), nous nous orientons vers le concept d'étayage que nous repérons chez la plupart de ces formateurs. En effet, selon Bruner, le processus d'étayage est une des composantes de l'interaction de tutelle faite par l'enseignant. Il consiste à "prendre en main ceux des éléments de la tâche qui excèdent initialement les capacités du débutant, lui permettant ainsi de concentrer ses efforts sur les seuls éléments qui demeurent dans son domaine de compétence et de les mener à terme " (Bruner, 1983). Ce concept est très proche de la notion de ZPD développée par Vygotsky, dont Bruner s'est inspiré. L'une des fonctions de l'étayage est le contrôle de la frustration. Il a pour finalité d'éviter que les erreurs de l'apprenant ne se transforment en sentiment d'échec, voire en une baisse de l'estime de soi telle que l'étudiant en viendrait à se considérer inapte à être infirmier. Cette préoccupation est apparue fréquemment et de façon prioritaire dans le discours des formateurs dont l'intervention de guidage est de nature étayante. Nous faisons l'hypothèse d'une particularité assez spécifique à la population de formateurs infirmiers. En effet, le fondement du travail infirmier est le " prendre soin », dans une perspective humaniste, centrée sur la personne, fortement influencée par la pensée de Carl Rogers. Cette préoccupation est particulièrement affirmée dans les propos et apparait dans les attitudes des formateurs au cours des séances, se manifestant par une attention bienveillante vis-à-vis de l'apprenant. 


\subsubsection{Un guidage « didactique » centré sur l'objectif-obstacle} pédagogique, ce qui spécifie un guidage « didactique ». Le but est de confronter l'étudiant à un problème, quelle que soit la nature du problème, y compris un imprévu, pourvu que celui-ci fournisse matière à réflexivité dans le feu de l'action. Au cours de sa séance, Daniel joue le rôle du médecin appelé au téléphone. Le scénario met en situation trois étudiantes au chevet d'un patient dont l'état général se dégrade brutalement. L'activitécible est l'évaluation de l'état clinique [reconnaissance des prodromes d'un accident vasculaire cérébral (AVC)] et l'appel téléphonique au médecin. Daniel considère ne pas avoir obtenu suffisamment d'éléments pertinents et renvoie l'étudiante à une observation clinique complémentaire. Il ne veut pas mettre le trio d'étudiantes en difficulté mais il ambitionne de les pousser à aller plus loin dans leur réflexion parce qu'il sait qu'elles ont potentiellement les ressources pour le faire, ce qui justifie sa décision :

DANIEL : En fait elles avaient juste remarqué qu'il y avait un problème au niveau de l'œil. Et j'attendais qu'elle me le donne dans son... dans son bilan. Et comme elle me l'a pas donné, je me suis demandé «comment on peut lui faire raccrocher en pointant... ? »... Parce qu'elle a commencé à faire une évaluation, elle avait demandé de serrer les mains, donc elle avait déjà un élément. Elles avaient regardé... au niveau du regard, elles avaient déjà vu qu'il y avait un problème au niveau des yeux. Donc j'ai deux éléments d'évaluation de conscience. [...] Et du coup, je me suis dit " comment je peux leur faire compléter son bilan?", sachant qu'elles avaient les informations, qu'elles avaient des éléments plus concrets à me donner...

$\mathrm{CH}$. : Comment tu as dosé finalement, entre le fait de les aiguiller et le fait de ne pas trop en dire?

DANIEL : Ben c'était... je savais que théoriquement, elles avaient vu cette approche et qu'elles l'avaient déjà abordée parce que c'était un des TD où on avait étudié le Glasgow $^{4}$. Et je me disais « il manque pas grand-chose! ». Bon, j'ai dit ben là, c'est un bon détonateur assez facile parce qu'elle sait... elle a déjà des données, elle a juste à être poussée pour qu'elle puisse faire le lien.

Cette volonté de challenger l'étudiant à la mesure de ses possibilités, de sorte qu'il puisse agir et apprendre, se traduit par un guidage mixte, étayant et didactique, privilégiant une stratégie combinant soutien et challenge, pour l'amener à dépasser ses routines de fonctionnement face à l'obstacle. Comme Daniel, Paula se situe dans une régulation plutôt didactique. Lors d'un scénario similaire, elle commente la manière dont, en tant que médecin appelé au téléphone, elle a renvoyé les étudiants à une observation complémentaire. Son intention est de les amener à se questionner en cours d'action et cette intention se conjugue avec celle de les préparer à ce qu'ils vont rencontrer dans la « vie réelle» :

PAULA : L'idée, c'est qu'ils découvrent par eux-mêmes, les étudiants, enfin qu'ils identifient justement « oh ben tiens, j'ai appelé trop tôt, quoi ! », et que finalement, s'ils vont pas recueillir les informations, leur appel va pas forcément être crédible. Et puis même dans la réalité, tu appelles le médecin, il va te dire «ben ouais, et alors? ", il faut bien qu'ils les récoltent les données! Or, si tu leur donnes tout de suite, ils vont dire «ben tiens, ça fonctionne, nickel!». Donc à la fois qu'ils réfléchissent aussi par eux-mêmes et qu'ils se rendent compte que quand tu fais un appel à l'aide, faut quand même que tu aies des éléments.

Le guidage didactique répond aussi à une stratégie concertée entre les formateurs. Nous avons dit que cette activité était conduite en binôme compte-tenu des multiples tâches à gérer. Paula explique la stratégie co-construite, et nous remarquons à cette occasion que 
le formateur oriente l'action en regard des objectifs d'apprentissage visés pour le débriefing :

PAULA : On a décidé que celui qui faisait le débrief, c'est celui qui faisait le médecin ou l'infirmier, parce que c'est vraiment lui qui est facilitateur à ce moment-là, et c'est lui qui va vraiment orienter... [...] Parce que de toutes façons, celui qui fait le patient, il rentre dans son rôle d'acteur, et il est concentré à fond dedans, donc il peut pas prendre du recul. La partie technique elle peut être gérée par celui qui va débriefer, ou par le patient - parce que c'est pas une partie technique très, très compliquée - mais ce qui est sûr que c'est que celui qui fait le patient c'est pas celui qui débriefe. Et je trouve que c'est bien que celui qui débriefe fasse médecin ou infirmier parce que c'est lui qui donne le ton, il sait où il veut aller.

\subsubsection{Un guidage « prospectif » visant l'obtention de matière pour le débriefing}

Comme nous le percevons dans le discours de Paula - et ce sera le cas pour la majorité des formateurs - obtenir des éléments significatifs en vue de les exploiter au cours du débriefing est une préoccupation importante, qui reste en toile de fond de leur observation et qui oriente leurs interventions. D'ailleurs pour certains, l'activité de guidage est prioritairement ciblée vers l'obtention de ce matériau, alors que pour d'autres (nous l'avons vu chez Luc), c'est une préoccupation secondaire. La logique qui prévaut dans le guidage prospectif est une logique pédagogique dans la mesure où ces formateurs considèrent le débriefing comme le moment où se construit l'apprentissage. Le guidage est prospectif car il vise à repérer et à produire le matériau expérientiel support :

MARC : Là, je me concentre sur la scène jouée, mes éléments de débriefing, et à quel moment je vais arrêter, à quel moment j'aurais suffisamment de matière pour pouvoir débriefer.

À l'extrême, l'activité des étudiants ne doit pas perturber le scénario, en l'occurrence faire que celui-ci puisse se dérouler comme prévu afin de pouvoir débriefer ensuite le contenu souhaité. Quelles que soient les difficultés repérées chez ces derniers, quelques formateurs interviendront comme ils l'ont planifié, tout en essayant de maintenir l'étudiant dans « les rails»:

YVES : Faire en sorte que nos situations elles puissent rouler... et surtout qu'on ait de la matière après pour le débriefing, quoi. [...] Nous, de toutes façons, on part du principe que notre situation elle part d'un point $\mathrm{A}$ pour aller à un point $\mathrm{B}$, que nous on a décidé. À la limite, quoi que fasse l'étudiant on y arrivera au point $\mathrm{B}$. Il y a ce rôle de facilitateur pour essayer de remettre dans les rails. Mais on n'arrive jamais à un point $C$, voilà. Donc nous, il va toujours au point $B$ que nous on a décidé.

Pour ce formateur, l'activité simulée n'est qu'un prétexte pour discuter plutôt la situation sur un plan généraliste. Cette pratique a été évoquée par Horcik (2014) qui a mis en évidence que l'objet des échanges lors du débriefing n'était pas tant l'analyse de l'activité des apprenants au cours de la situation simulée, que le parallèle possible avec l'activité réelle de travail. Ici, le mode d'intervention d'Yves est plutôt ciblé sur la situation, quels que soient les besoins des apprenants. Ce que vise ce formateur est davantage la discussion, au cours du débriefing, sur la reconnaissance des signes d'un AVC en référence à la connaissance du processus physiopathologique et sur l'appel à l'aide selon une procédure standardisée. Il focalisera peu sur l'analyse de l'activité singulière de l'étudiant. Le mode de guidage est donc prospectif: la situation et l'activité de l'apprenant ne sont qu'un prétexte au débriefing, et à l'extrême, peu importe ce qu'il s'y est passé. 


\subsubsection{Un guidage « de commodité » influencé par son propre degré de confort dans l'exercice}

Le guidage prospectif est parfois relié à un guidage dit " de commodité ». Ce dernier est ciblé sur le formateur et destiné à le maintenir dans sa zone de confort, lui permettant d'aller là où il a prévu d'aller, quoi qu'il se passe au cours de l'exercice, comme nous l'avons vu chez Yves. Fauquet et Frémaux (2008, cités par Vidal-Gomel et al. 2011, p. 120), à l'occasion d'une étude portant sur la formation des médecins anesthésistes, évoquent chez les formateurs l'évitement de la "situation inacceptable » (le décès du patient), non pas tant pour préserver les stagiaires d'un sentiment d'échec douloureux, que pour se préserver soi-même d'avoir à gérer les conséquences psychologiques d'une telle situation.

Dans notre étude, la préoccupation relative au débriefing est associée à un autre type de crainte : celle de manquer de matériau expérientiel. En effet, contrairement à Luc et à quelques formateurs expérimentés qui jugent qu'ils auront matière, quoi qu'il se passe, plusieurs formateurs - les plus novices mais pas seulement - se sentent démunis lorsque les étudiants vont trop vite pour résoudre le problème posé. Le guidage vise alors à orienter la situation vers ce que le formateur maitrise et à le préserver d'une trop grande incertitude ou d'une "peur du vide", ainsi que l'expriment certains d'entre eux. Ici, s'exprime une logique de sécurisation.

Nous l'illustrons avec la séance d'Anne. Après le briefing expliquant le contexte de la prise en charge du malade (patient douloureux, dans une problématique de pathologie cancéreuse, sous morphine en perfusion continue), trois étudiantes entrent en action. Celles-ci, d'après les symptômes et l'état de conscience du patient (nausées, somnolence, bradypnée,...), identifient très vite le problème de santé (un surdosage en morphine). Elles prennent des mesures correctives (arrêter la perfusion, surélever la tête du patient) et appellent ensuite le médecin en ayant pris soin de recueillir des données cliniques objectives. Elles démontrent ainsi une prise en charge tout à fait adaptée. Anne a le rôle du médecin appelé au téléphone. Déstabilisée par la crainte de ne pas avoir assez de « matière » à débriefer, elle va profiter d'un dysfonctionnement pour ajouter un obstacle (Tableau 2): 
Tableau 2 : Extrait situation simulée et autoconfrontation d'Anne.

Table 2: Extract from simulated situation and post situation interview of Anne

\begin{tabular}{|c|c|}
\hline EXTRAIT SITUATION SIMULEE - ANNE & EXTRAIT ENTRETIEN D'AUTO CONFRONTATION - ANNE \\
\hline $\begin{array}{l}\text { ANNE : Allo, oui, Dr Martin au téléphone, c'est pour } \\
\text { quoi ? } \\
\text { ESI : Je vous appelle au sujet de Mr Belin. Qui, } \\
\text { apparemment fait une réaction à la morphine. Il a } 9 \\
\text { de tension, il a } 99 / 68, \text { euh... } 66 \text { de pouls...euh... il se } \\
\text { sent pas bien, il a envie de vomir. Alors ce qu'on a } \\
\text { fait, c'est qu'on a arrêté la perfusion de morphine. } \\
\text { Et... pour l'instant c'est tout ce qu'on a fait... } \\
\text { ANNE : Oui... d'accord, OK... } \\
\text { ESI : ... Voilà, c'est tout ce qu'on a fait pour l'instant. } \\
\text { ANNE : ... II l'avait depuis combien de temps, la } \\
\text { morphine ? } \\
\text { ESI : ... Humm... alors la morphine... il l'a depuis... } \\
\text { (elle se tourne vers sa collègue) la morphine il l'a } \\
\text { depuis quand ? ... (au médecin) excusez-moi, hein, } \\
\text { je regarde dans son dossier... ah... c'est pas } \\
\text { marqué... } \\
\text { ANNE : Ça a été prescrit quand? } \\
\text { ESI : Ça a été prescrit... ah, il y a pas de date sur la } \\
\text { prescription... } \\
\text { ANNE : Bon, d'accord..., Je vais passer. En attendant } \\
\text { essayez de prévoir l'antidote. } \\
\text { ESI : ... Oui... } \\
\text { ANNE : OK, merci. (Elle raccroche) }\end{array}$ & 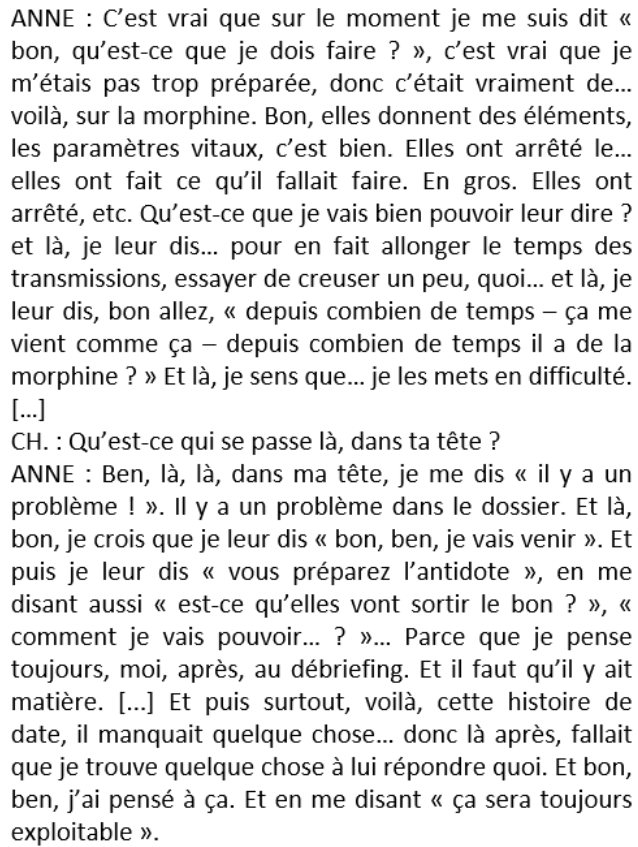 \\
\hline
\end{tabular}

Dans cet extrait, nous voyons qu'Anne est prise au dépourvu face à la bonne gestion de la situation par les étudiantes; elle n'a manifestement pas envisagé ce cas de figure: " qu'est-ce que je vais bien pouvoir leur dire? ». Or, le contexte requiert qu'elle réagisse tout de suite : il lui faut prendre une décision sur la manière d'interagir avec les étudiantes en tant que médecin au téléphone. C'est alors que survient un aléa : l'absence de date sur la prescription médicale. C'est un oubli de la part des formateurs de la séance de la veille qui n'ont pas réactualisé le dossier-patient. Anne explicite comment elle transforme alors l'aléa - non intentionnel mais pouvant être crédible sur le plan du réalisme - en opportunité d'apprentissage, en projetant d'évoquer la règlementation relative à la prescription médicale : «ça sera toujours exploitable ». Par ailleurs, la conduite tout à fait pertinente des étudiantes lui fait craindre de ne pas avoir assez de matériau à débriefer : "Parce que je pense toujours, moi, après, au débriefing. Et il faut qu'il y ait matière ». Anne opère alors un guidage tout autant "prospectif " que "de commodité », destiné à obtenir davantage de matière à débriefer et à se sentir plus à l'aise dans le débriefing. Nous repérons à cette occasion que sa conception de l'apprentissage ne lui permet pas d'envisager une pratique conforme comme source d'apprentissage suffisante, mais que c'est pour elle davantage l'erreur ou l'écart entre un idéal de bonne pratique et la pratique observée qui seraient apprenants. Anne se sent plus à l'aise lorsqu'il y a matière à travailler sur les écarts. Mais, ce faisant, elle s'inscrit aussi dans un mode de guidage didactique, voulant corser la difficulté chez des étudiantes capables d'aller au-delà de l'obstacle initial. Cependant, ses propos montrent qu'il s'agit ici davantage d'une improvisation faite sur le vif et destinée à la sortir d'une situation insécurisante pour ellemême, qu'une stratégie pédagogique réfléchie et anticipée. 


\subsubsection{Un guidage « normatif » ciblé sur le référentiel prévu}

lorsque les potentialités de certains étudiants pourraient leur permettre d'aller au-delà de ce qu'ils avaient envisagé. Ils considèrent que le scénario planifié doit se dérouler tel quel, au risque que la situation - comportant déjà par nature des éléments imprévisibles ne leur échappe si d'autres obstacles sont introduits. Ainsi, dans la séance animée par Rose, un étudiant est confronté à une patiente manifestant une anxiété majeure en lien avec la perspective d'une chirurgie pour un cancer du sein. Cette anxiété se manifeste par une humeur labile, entre révolte, peur et détresse. Après avoir réussi à apaiser la patiente, l'étudiant commence à instaurer une véritable relation de soutien avec elle. Pourtant, Rose met fin au scénario car ce qui a été décidé par le collectif de formateurs, c'est de stopper la simulation lorsque la patiente a pu exprimer les trois registres d'humeur qui étaient prévus. Ici, l'objectif visé est en deçà des capacités de l'étudiant. Celui-ci semblait en effet en mesure de dépasser le premier niveau de conduite d'entretien (reconnaitre les manifestations du processus de perte et adapter son mode relationnel) puisqu'il s'apprêtait à s'engager dans une relation d'aide, activité d'un niveau de complexité supérieur. Rose n'aura quant à elle aucun mal à conduire le débriefing en mettant l'accent sur la conduite tout à fait adaptée de l'étudiant. Pourtant, lors de son entretien d'autoconfrontation, elle s'interroge :

ROSE : Après, peut-être le scénario... est-ce qu'on aurait pu aller un petit peu plus loin? Parce que les étudiants m'ont dit qu'ils étaient frustrés. Et peut-être qu'effectivement en allant un petit peu plus loin dans le scénario, on aurait peutêtre pu mettre au travail certains concepts qu'on avait abordés pendant la semaine et du coup aller un petit peu plus loin dans cette situation de soins relationnels. Donc ça, effectivement, je vais le confronter avec mes collègues pour voir ce qu'ils en pensent, mais peut-être que ce scénario, on peut l'enrichir un peu, quand on arrive après la phase trois, où la personne accepte d'entrer en relation. Parce que là, ouais, du coup, j'ai senti que ça les avait frustrés.

Or, dans les autres groupes observés, certains étudiants n'ont pas été en mesure d'aller au-delà de ce premier niveau de relation. Les objectifs visés étaient donc adaptés aux capacités du public d'étudiants. Cependant, ces formateurs ne s'autorisent pas ici à aller plus loin lorsqu'un étudiant en manifeste le potentiel. Ils le justifient par un souci de cohérence intergroupes (voire d'«équité») et d'harmonisation des pratiques interformateurs, préoccupation revenant régulièrement dans leurs discours (quatre d'entre eux exercent ensemble dans l'Ifsi 3). Nous identifions ici un mode de guidage que nous qualifions de «normatif », dans une logique de conformité au référentiel prévu.

\subsubsection{Un guidage « productif » ciblé sur le réalisme de la situation}

Pour deux formateurs seulement, la centration des interventions de guidage porte prioritairement sur la situation plutôt que sur l'activité des étudiants. Nous qualifions ce guidage de "productif ", en référence aux travaux de Wagemann et Percier (1995) et de Cardin (2016), op. cit. : l'intervention de guidage est ciblée sur les variables de la situation, avec une orientation dominante vers la recherche de fidélité à la situation réelle de référence et la crédibilité des interventions. La logique est ici celle de l'expert-métier.

$\mathrm{Au}$ cours de la séance animée par Franck, les étudiants doivent repérer des signes cliniques péjoratifs indiquant que le patient est en train de faire un AVC, et appeler le 
médecin. Franck est dans la salle de débriefing et joue le rôle du médecin au téléphone. Il commente sa manière de faire, inspirée de sa propre expérience en tant qu'infirmier pour donner du réalisme à son intervention (Tableau 3) :

Tableau 3 : Extrait situation simulée et autoconfrontation de Franck. Table 3: Extract from simulated situation and post situation interview of Franck

\begin{tabular}{|c|c|}
\hline $\begin{array}{l}\text { EXTRAIT SITUATION SIMULEE - } \\
\text { FRANCK }\end{array}$ & EXTRAIT ENTRETIEN D'AUTO CONFRONTATION - FRANCK \\
\hline $\begin{array}{l}\text { FRANCK (décrochant le téléphone): } \\
\text { Oui, Docteur Privat, bonjour... } \\
\text { ESI : oui, c'est l'étudiante infirmière du } \\
\text { service de médecine, j'ai Mr Lambert, } \\
\text { un patient qui était là pour des } \\
\text { céphalées, et sa femme a voulu qu'il } \\
\text { soit hospitalisé... } \\
\text { FRANCK : oui ? } \\
\text { ESI : ... il a des antécédents d'AVC en } \\
\text { mai } 2014 \text { et il répond plus là... } \\
\text { FRANCK : il répond plus, c'est-à-dire ? } \\
\text { ESI : euh... ben il a une perte de } \\
\text { conscience, il répond pas... il a pas de } \\
\text { réponse motrice } \\
\text { FRANCK : Il est inconscient, là ? } \\
\text { ESI : euh... (elle se tourne vers ses } \\
\text { collègues) il est conscient? } \\
\text { FRANCK (impatient) : allo ?! } \\
\text { ESI : non il est... } \\
\text { FRANCK (I'air agacé) : il est inconscient } \\
\text { ou pas ?! ... Je vous entends mal! } \\
\text { ESI : (hésitante, se retourne vers ses } \\
\text { collègues) Les filles, dites-moi si... } \\
\text { FRANCK : Bon écoutez, là, j'ai pas trop } \\
\text { le temps, là. Vous essayez d'évaluer s'il } \\
\text { a des troubles de la conscience et vous } \\
\text { me rappelez. }\end{array}$ & $\begin{array}{l}\text { CH. : Qu'est-ce qui a guidé ta... ton action, ton discours ? } \\
\text { FRANCK : Ben mon expérience. Voilà. } \\
\text { CH. : Ton expérience ? } \\
\text { FRANCK : Mon expérience professionnelle d'infirmier de service } \\
\text { d'urgence, de réanimation. [...] L'intonation du médecin pas très } \\
\text { patient, qui a besoin tout de suite d'informations. Donc je pense } \\
\text { sans prétention que c'est tout à fait le reflet de ce que, moi, j'ai pu } \\
\text { entendre des dizaines de fois. } \\
\text { CH. Quelle était ton intention dans cette interaction que tu as eue } \\
\text { là pendant la situation ? } \\
\text { FRANCK : Alors il y a deux choses. C'est d'abord l'importance de } \\
\text { l'observation clinique. Quand je renvoie concrètement « ben il est } \\
\text { inconscient ou il a des troubles de la conscience ? ". Et puis la } \\
\text { deuxième chose, c'est l'approche plus de communication et la } \\
\text { posture, le discours que doit avoir l'infirmier quand il transmet un } \\
\text { message... d'alerte, en l'occurrence. Donc là il y a deux objectifs. II } \\
\text { y a l'observation et le raisonnement clinique. Et euh... la } \\
\text { pertinence du message transmis. } \\
\text { CH. : Et concrètement comment ça s'est traduit dans ton... dans ta } \\
\text { façon de... d'être en interaction avec les étudiants? } \\
\text { FRANCK : Ben j'ai exprimé pas mal d'impatience... et encore j'ai été } \\
\text { très correct... parce qu'il s'agit pas non plus de les casser. Mais le } \\
\text { médecin est impatient, il veut avoir les informations de suite pour } \\
\text { savoir à quoi il a à faire. } \\
\text { CH. : Donc c'est plus finalement la réalité... enfin la fidélité à la } \\
\text { réalité de la situation qui t'a guidé.... c'est ça? } \\
\text { FRANCK : Oui, oui. Clairement. }\end{array}$ \\
\hline
\end{tabular}

68 Si Franck vise la confrontation de l'étudiant à l'obstacle, dans un mode de guidage didactique, c'est davantage pour l'entrainer à ce qu'il va vivre en situation réelle que dans une perspective de conceptualisation de l'action au cours du débriefing: pour lui, l'apprentissage se fait prioritairement dans l'action (il fera souvent référence à la nécessité de "rendre l'enseignement "palpable" et regrette la disparition des MSP »). Pour Franck, la mise en situation prime sur le débriefing, celui-ci ne servant qu'à fixer l'apprentissage expérientiel. La conception de l'apprentissage qui prévaut ici est la croyance que plus l'environnement est reproduit de façon réaliste et plus le sujet se confronte à des difficultés (voire à l'erreur), plus l'apprentissage opère. Ayant renvoyé les étudiantes à une observation clinique complémentaire, celles-ci se retrouvent en difficulté avec un matériel qu'elles ne savent pas utiliser (la fonction "prise de tension artérielle » sur le moniteur de surveillance cardiaque). Cet obstacle, qu'elles ne semblent pas en mesure de dépasser, nécessiterait en principe l'intervention d'un facilitateur parce qu'il les empêche de gérer le problème. Franck observe la scène mais n'intervient pas. L'assistant technique vient le voir pour lui demander quoi faire, mais Franck décide de ne pas intervenir. À cause de cette difficulté, la situation s'enlise et cela dure plusieurs minutes. À un moment, l'assistant technique ira de son propre chef montrer aux étudiantes comment utiliser le monitoring cardiaque pour afficher la prise de tension artérielle. Lors de l'entretien, nous revenons sur cet incident.

CH. : Tu n'es pas intervenu dans la situation. Est-ce que tu peux m'expliquer pour quelles raisons? 
FRANCK : Ben des fois il peut y avoir un intérêt aussi à pas réagir tout de suite...

CH. : C'est-à-dire?

FRANCK: C'est-à-dire que la capacité d'adaptation... ce qu'on dit sur le plan théorique, ben concrètement elle est là. Sur le plan pratique. Comme dans la réalité de service on n'a pas, et c'est souvent le cas, tout, tout de suite, donc il faut s'adapter... à pouvoir résoudre des problématiques... techniques en l'occurrence. Je me souviens plus trop mais il y avait peut-être ce genre d'objectif... est-ce qu'ils sont en capacité de se débrouiller? ... Alors pas seuls mais en étant en équipe. D'interagir pour être en mesure de résoudre des problèmes.

69 Là encore, c'est la fidélité à la situation réelle qui guide Franck dans sa manière de faire : il considère que l'obstacle technique (qui n'était pas intentionnel au départ) est source d'apprentissage parce que dans la «vraie vie », l'étudiant peut être confronté à de telles difficultés. C'est le point de vue essai-erreur qui prime pour lui, ce qui lui fait choisir d'utiliser cet obstacle imprévu, en n'intervenant pas. Chez Franck, le souci de maintenir les étudiants dans leur ZPD n'est pas présent : ces étudiantes, non familiarisées avec ce type de matériel spécifique des services de soins intensifs, n'étaient pas en capacité de l'utiliser.

\subsubsection{Au total, un guidage multimodal}

70 Observer les formateurs dans l'action et surtout les écouter expliciter ce qui guide leur intervention, nous permet d'affirmer que leur activité cognitive au cours de la situation simulée est intense. Leurs interventions de guidage répondent à des préoccupations de différentes natures, qui doivent être simultanément examinées, évaluées et concrétisées. Plus ils sont expérimentés, plus le registre de guidage est élargi vis-à-vis des différentes cibles, traitées simultanément. Ces formateurs observent l'activité de chacun des protagonistes dans ses multiples dimensions. Ils analysent, soupèsent les options possibles pour la suite, décident d'intervenir ou pas, adaptent leur guidage aux différents objectifs poursuivis et recueillent le matériau le plus pertinent en vue du débriefing. La plupart ajustent leur manière d'agir à des préoccupations étayantes et éthiques: maintenir l'étudiant dans sa ZPD et le garder en sécurité, en l'occurrence ne pas le mettre en échec sous le regard de ses pairs. Par ailleurs, ils restent ouverts à l'émergence d'éléments imprévus qui peuvent constituer des opportunités d'apprentissage, ainsi qu'en témoignent les propos de cette formatrice experte :

VIVIANE : Quand c'est le mannequin, on parle et on observe en même temps, donc ça veut dire qu'il faut être au clair avec ce qui se passe dans la situation pour réagir de façon opportune et donner les indices pertinents au bon moment, et adapter à chaque fois à chaque étudiant, ou à chaque binôme ou chaque trinôme. Parce que ceux qui sont en difficulté, je leur donnerais peut-être plus d'indices parce que mon intention est qu'ils s'en sortent relativement satisfaits... alors c'est pas toujours le cas, mais qu'au moins ils réussissent à faire quelque chose... Et puis les groupes qui s'en sortent plutôt bien, ben je vais pas donner trop d'indices, je vais même peut être les mettre en difficulté sur deux ou trois points supplémentaires... donc c'est à adapter... En même temps, c'est repérer les moments-clés qui vont être en lien avec les axes de débriefing et puis éventuellement des axes potentiels que tu avais pas du tout envisagés mais qu'il faut absolument reprendre parce qu'il y a un problème que tu as identifié dans la situation.

71 Dans le même temps, les formateurs s'autorégulent en contrôlant leur propre stress, relatif à la charge mentale requise pour eux-mêmes dans cet exercice. Ils développent des stratégies d'adaptation plus ou moins efficaces, selon leur degré de confort et la confiance qu'ils ont en leur capacité de faire face aux exigences de la situation didactique. C'est un 
exercice particulièrement stressant pour les formateurs novices, même si, comme Élise, certains ont une longue expérience de la formation :

ELISE : Je pense quand même que... qu'il faut avoir suffisamment de confiance en soi pour pouvoir aborder ça... euh... je dis en live, parce que c'est ça. Parce qu'on sait pas ce qu'on va avoir. [...] Ben j'en ai pas conduit beaucoup, donc là, je suis très angoissée pour les prochaines séances. Je suis morte de trouille en fait!

Sandra, jeune formatrice, est en revanche plutôt confiante; elle réinvestit dans cet exercice des compétences acquises lors de son expérience comme infirmière de réanimation: une vision globale de la situation, une capacité d'anticipation lui permettant de pouvoir gérer plusieurs activités simultanément.

SANDRA : La réa m'a appris à avoir cette vision périphérique de «je vérifie si tout va bien et puis après je me jette à l'eau » quoi. Et je pense que c'est ça que j'ai fait, du coup... à mon avis, ça a dû m'aider ce travail d'infirmier là... tu vois ? Parce qu'en réa, tu es obligé quand même d'avoir... tu as le côté technique, le côté relationnel, tu as le côté soins, faut que tu sois bon dans les soins, et tu peux faire du coup pas mal de parallèle avec notre activité en simulation, pour le coup!

$\mathrm{Au}$ fur et à mesure, les formateurs les plus expérimentés parviennent à composer avec l'incertitude et à construire un répertoire d'interventions, ainsi que nous l'avons vu supra avec Sylvie l'experte :

XAVIER : On a des... entre guillemets, des imprévus... qui ne sont quasiment plus des imprévus, compte-tenu de notre recul... c'est-à-dire qu'on arrive même parfois à anticiper en se disant « ouh, il va y avoir un problème, là ! "

Ils capitalisent ainsi un répertoire de pratiques qu'ils partagent généralement au sein du groupe de pilotage. Selon que ce collectif est plus ou moins ouvert, les autres collègues vont pouvoir en profiter. Dans l'Ifsi 3, les formateurs sont très soucieux d'harmoniser leurs pratiques et se situent dans un mode de guidage plutôt normatif qui englobe des modes de guidage étayant et didactique. Dans les deux autres Ifsi, le partage d'expérience a posteriori des séances est moins développé, et les responsabilités sont plus cloisonnées (nous l'avons vu avec le briefing des comédiens fait par le chef du groupe de pilotage); nous retrouvons alors des profils un peu plus hétérogènes en ce qui concerne les cibles privilégiées du guidage.

\section{Discussion : un agir en tension dans un entremêlement de préoccupations}

75 Nous avons tenté de débusquer les préoccupations qui sous-tendent l'activité du formateur dans cette fonction de guidage assez complexe. À l'instar de Vidal-Gomel et al. (2011), nous considérons celle-ci comme un processus de régulation en tension entre plusieurs préoccupations qui doivent être simultanément prises en compte. Chaque élément de la situation produit un effet, à l'image d'une réaction en chaine, et chaque effet devient une nouvelle préoccupation à analyser et à traiter. En conséquence, les modalités de guidage sont plurielles et interdépendantes. D'un côté, il s'agit de rester dans le référentiel prévu afin de garantir un travail relativement homogène en termes d'objectifs pédagogiques, fixés ex ante pour tous les groupes d'étudiants. De l'autre, il existe des initiatives, délibérées ou non, volontaires ou contraintes, pour utiliser l'aléa ou l'imprévu en vue d'atteindre de nouveaux objectifs. Le guidage normatif est donc parfois en tension avec le guidage didactique. Dans le même temps, il s'agit de trouver un compromis entre la nécessité de challenger l'apprenant et celle de lui garantir du soutien 
et une sécurité psychologique, tout en s'assurant à soi-même un certain degré de confort personnel ou de maitrise dans l'activité. Ainsi, le guidage didactique est souvent en tension avec le guidage étayant et parfois avec le guidage de commodité, amenant le formateur à doser plus ou moins habilement la prise de risque, tant pour l'apprenant que pour lui-même. Il s'agit aussi de gérer le temps présent de l'exercice simulé et de préparer le temps suivant, dont le contenu est soumis à ce qui sera produit en situation. Ainsi, il convient de conduire la situation dans un souci de crédibilité clinique et de fidélité à la situation de travail, tout en la considérant dans sa dimension didactique, comme situation de formation, autorisant par là des distorsions par rapport au réel, et tout en assurant l'obtention de matière pertinente pour le débriefing. Il y a alors tension entre guidage productif, guidage didactique et guidage prospectif. Les préoccupations des formateurs sont, en ce sens, des préoccupations entremêlées, et les logiques d'action sont mixtes.

Ces préoccupations, orientées chacune vers une cible spécifique, sont en perpétuelle reconfiguration et recomposition en cours d'action, dans une temporalité brève. Les modalités de guidage qui leur répondent sont toutes présentes, à des degrés variables, chez chacun des formateurs rencontrés, mais certaines sont privilégiées - voire dominantes. Pour la majorité d'entre eux (souvent les plus expérimentés en pédagogie), ce sont les modalités étayante, didactique et prospective qui dominent, dans une logique pédagogique : leur ambition est d'abord de former des professionnels réflexifs, dans une perspective développementale de l'apprentissage. Les novices en revanche (mais pas tous !) - ou ceux qui, malgré l'expérience, ressentent encore un stress important dans cet exercice - ont un mode de guidage plus normatif et de commodité, témoignant d'un besoin de repère et de conformité à la prescription, et ils prennent en compte moins de cibles à la fois. Mais la plupart d'entre eux manifeste un guidage étayant qui privilégie le soutien à l'apprenant au détriment parfois de l'atteinte des objectifs visés ou de la fidélité à la situation réelle de référence. Ceux, expérimentés ou moins, pour lesquels le collectif de pairs fonctionne selon un mode collaboratif davantage que coopératif (Ifsi 3) ont un mode de guidage plutôt normatif, visant le respect des orientations prises par le collectif de formateurs. Ils ont pourtant développé le guidage didactique, quitte parfois à s'affranchir de certains choix collectifs. Les formateurs qui se revendiquent « infirmiers avant d'être formateurs » (et certains ont pourtant de nombreuses années d'expérience dans la formation) ont un mode de guidage plus productif que didactique, recherchant la fidélité à la situation de référence : leur but est d'abord de former des professionnels opérationnels, et leur conception de l'apprentissage privilégie l'apprentissage par l'erreur, très présent dans leur discours. Ce dernier point semble contredire les résultats mis en évidence par Wagemann et Percier (1995) qui montraient des formateurs « issus $\mathrm{du}$ rang » orientant l'action vers sa réussite; nous pourrions expliquer cela du fait du caractère particulièrement tabou de l'erreur en médecine, et de l'opportunité qu'offre la simulation d'apprendre en pouvant "se tromper sans risque pour le patient ", leitmotiv largement mis en avant par les pouvoirs publics et par les sociétés commerciales vendant des mannequins. Cela concerne cependant un petit nombre de formateurs.

Ces sept modalités peuvent être représentées schématiquement, avec leur cible privilégiée, sous la forme d'une rosace, entremêlant ses pétales (Figure 3). Le positionnement $\mathrm{du}$ formateur dans ces différentes logiques est un positionnement dynamique, influencé par le contexte mouvant de la situation simulée et de ses exigences, par ses propres croyances en matière d'enseignement, par ses propres capacités de composer avec l'incertitude et de se situer dans cet environnement instable, prenant en 
compte les besoins de l'apprenant, les normes du groupe de pairs et de l'institution, et ses propres buts.

Figure 3 : Modalités de guidage en tension dans un entremêlement de préoccupations chez le formateur au cours de la situation simulée.

Figure 3: Modalities of guidance in tension within integrated preoccupations by the trainer during the simulated situation

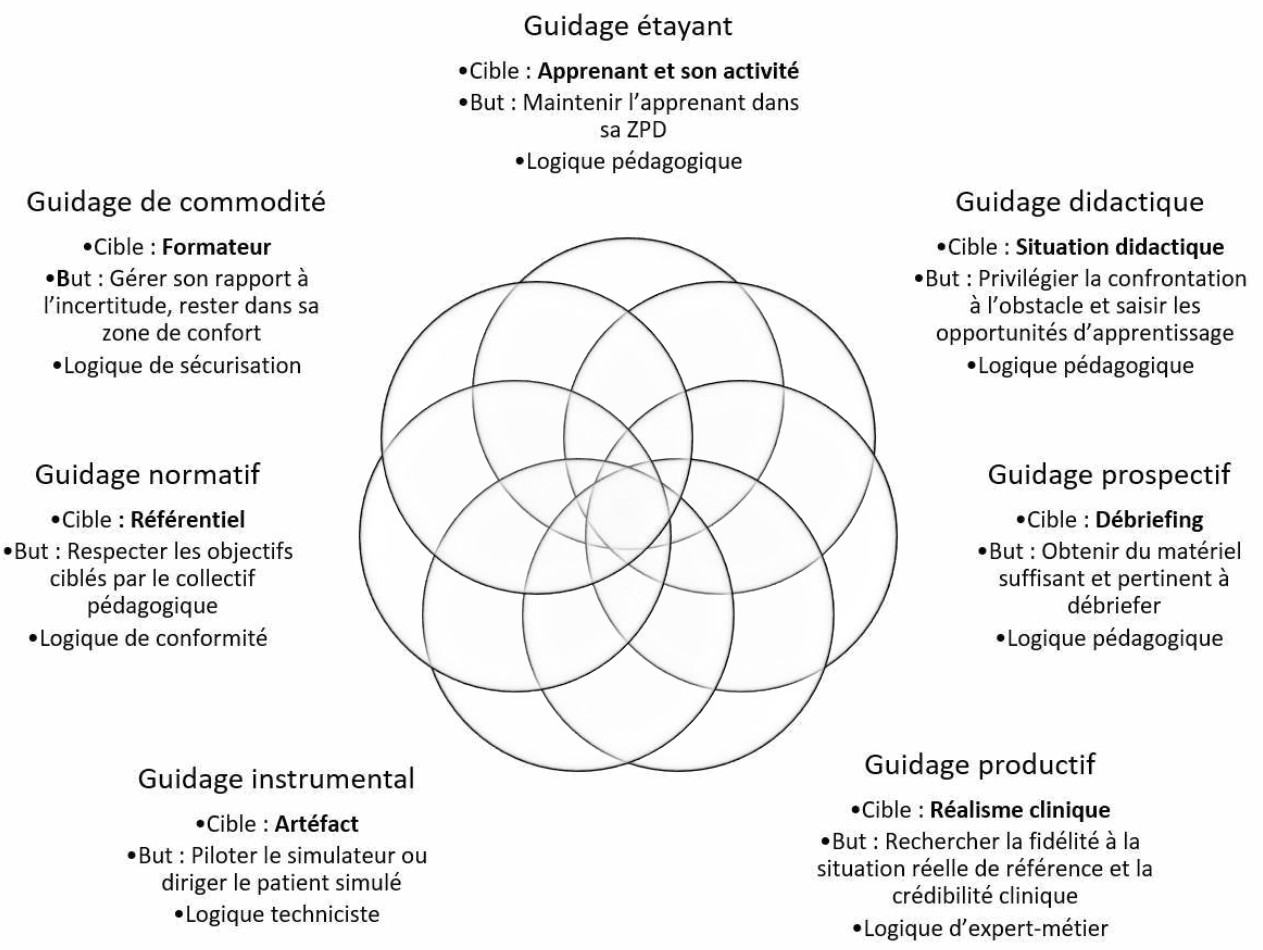

Cet entremêlement n'est pas sans rappeler les travaux de Bucheton et Soulé (2009) évoquant l'empan des gestes d'ajustement de l'enseignant dans un multi agenda de préoccupations enchâssées : "l'empan est l'ensemble des variables qu'il [l'enseignant] prend en compte pour interpréter et agir en situation, la manière dont il configure ses différentes préoccupations, dont il accentue ou focalise son attention sur certaines d'entre elles et ceci de manière régulière ». Dans cet exercice, l'empan est particulièrement large, compte-tenu que, contrairement à d'autres formats pédagogiques plus traditionnels, le formateur ne dispose pas d'une trame préétablie relative à un contenu à dispenser, puisqu'il travaille ici à partir du matériau «en train de se faire » et qui comporte une part importante d'imprévisibilité. Il est probable que, dans ce contexte incertain, le formateur navigue entre ces sept préoccupations, composant plus ou moins habilement entre plusieurs d'entre elles, ou privilégiant sciemment ou parfois à son insu les unes ou les autres en fonction du contexte singulier d'une séance et de ses déterminants (type de scénario et d'artéfact, dynamique du groupe d'apprenants, nature de la collaboration avec le binôme, etc.) et de sa propre aisance dans l'exercice.

Cependant, nous pensons que, régulièrement, la manière dont les formateurs opèrent dans cet exercice d'équilibriste traduit leur sentiment de confiance en soi, leurs valeurs, leurs croyances relatives au processus d'apprentissage, et comment ils ont intégré les dimensions pédagogiques et didactiques de leur métier. Elle traduit aussi probablement leur imprégnation des pratiques locales. Si ces éléments colorent la manière dont ils vont privilégier le plus souvent une ou deux cibles en particulier, il semble que les formateurs 
tentent de faire des compromis les plus équilibrés possibles entre des couples de préoccupations, en fonction des différentes exigences de la situation dynamique quand celles-ci leur paraissent contradictoires.

80 Ainsi, ceux qui privilégient un guidage normatif peuvent parfois s'autoriser à sortir du déroulement prévu lorsque leur analyse de la situation détecte une opportunité nouvelle. Ce compromis sera d'autant plus acceptable que le collectif de pairs considèrera la prise d'initiatives possible, pertinente et profitable pour les étudiants, et dans la mesure où eux-mêmes se sentiront capables de prendre cette liberté. L'équilibre se construit alors en parvenant à composer avec souplesse entre un certain degré d'autonomie par rapport au référentiel prescrit (quitte à sacrifier certains objectifs prévus) et la nécessité d'inscrire son action en cohérence avec les orientations décidées par le collectif (mais dont un cadrage trop strict leur ferait rater des opportunités d'apprentissage). C'est ce que Paula exprime ici :

«Je me sens... bien armée, maintenant. Plus rien me fait peur, quoi, tu vois? Et puis

du coup, je fais vraiment à ma sauce, sans jamais sortir du cadre ».

81 Par ailleurs, nous constatons que le fait d'être novice ou expert dans cette activité ne présage pas qu'un formateur manifestera telle ou telle priorité dans son guidage, même s'il est clair que le novice gère moins de cibles simultanément. En effet, nous remarquons qu'un formateur ayant pris ses fonctions depuis peu à l'Ifsi peut être très vite à l'aise dans cet exercice, compte-tenu de la proximité de celui-ci avec l'exercice infirmier de terrain (c'est le cas de Sandra qui se sent à l'aise dans l'incertitude du fait de compétences adaptatives acquises en réanimation). Ou bien qu'au contraire il a besoin de se référer à des normes très structurantes pour pouvoir agir. Il apparait aussi que certains formateurs expérimentés en pédagogie ont du mal à se libérer de leurs routines de fonctionnement mais que d'autres au contraire ont davantage de facilités à aborder l'incertitude avec la souplesse nécessaire, du fait de leur compréhension plus fine des différents enjeux pédagogiques et didactiques. Ces éléments indiquent clairement qu'il y a plusieurs chemins possibles pour parvenir à développer cette compétence. Il est donc nécessaire de s'interroger sur ce qui favorise l'élaboration de compromis équilibrés, celle-ci traduisant selon nous l'atteinte d'un bon niveau de compétence dans l'activité de guidage.

\section{Conclusion}

82 Notre contribution a présenté une typologie des modalités de guidage au cours de l'exercice simulé. Si le contexte singulier de la situation influence nécessairement la manière dont le formateur va opérer le guidage, nous pensons que des tendances comportementales sont retrouvées assez régulièrement chez les formateurs, en particulier chez les formateurs expérimentés qui ont construit des invariants opératoires de leur pratique. La capacité d'élaborer des compromis entre des buts contradictoires est un indice d'un niveau de compétence élevé et probablement d'un haut degré de confiance en soi chez les formateurs. Compte-tenu de la forte incertitude inhérente à ce format pédagogique, les dimensions d'autorégulation et d'ajustement sont une caractéristique particulière de leurs pratiques. En accord avec Huber et Chautard (2001), nous considérons que cette activité de guidage en situation simulée se situe dans une zone potentielle d'imprévus (ZPI) pour le formateur ; c'est-à-dire que la situation simulée est un espace où des imprévus vont survenir du fait de l'action des différents protagonistes (les étudiants, l'acteur, le(s) formateur(s)), même si, avec l'expérience, les formateurs 
développent une reconnaissance de plus en plus fine des réactions possibles des étudiants, et peaufinent ainsi leurs stratégies de guidage. Ici, une ZPI est suscitée, induite sciemment et volontairement par le formateur, afin d'introduire la confrontation à l'obstacle pédagogique; il s'agit donc d'un véritable espace d'imprévus. Prévoir et mettre en œuvre de telles zones dynamiques nécessite, de la part des formateurs, des précautions afin qu'il n'y ait pas de malentendus, en particulier que les étudiants ne ressentent pas cela comme un piège et que les deux formateurs (ou l'acteur-patient et le formateur) puissent construire entre eux une collaboration harmonieuse et cohérente.

Naviguer dans ces espaces d'imprévus n'est donc en aucun cas synonyme d'improvisation (Jean, \& Etienne, 2009) : c'est un espace qui a été pensé, réfléchi en amont afin d'envisager des stratégies d'adaptation aux diverses exigences des options possibles ou probables, et d'être ainsi en mesure d'élaborer des compromis acceptables et équilibrés. La dimension d'ingénierie des séances de simulation est donc particulièrement cruciale à développer, tout comme la réflexivité sur sa propre pratique en tant que formateur. D'une part, la connaissance des différents enjeux en lien avec les sept cibles identifiées peut aider les formateurs à conceptualiser et à étayer leurs pratiques en repérant mieux les ZPI. D'autre part, l'analyse de l'activité de guidage des formateurs nous parait particulièrement propice à l'identification des cibles dominantes, à la conscientisation des buts poursuivis et de la manière dont les compromis se forment. Cela pourrait sans doute s'opérer grâce à des dispositifs d'analyse de l'activité tels que l'autoconfrontation croisée (Clot, Faïta, Fernandez, \& Scheller, 2000), l'instruction au sosie (Clot, 2001) ou encore l'entretien d'explicitation (Vermersch, 1994). Ainsi, ces deux points devraient, à notre sens, relever d'une attention spécifique dans la formation des formateurs à la conception et à la conduite de séances de simulation, et dans leur accompagnement en situation professionnelle.

\section{BIBLIOGRAPHIE}

Argyris, C., \& Schön, D. (1996). Organizational Learning: Theory, Method, and Practice. Reading MA : Addison Wesley.

Boucheix, J.-M. (2015). Apprendre le réel sans les risques du réel ? L'apprentissage sur simulateur comme prévention. [En ligne] https://www.researchgate.net/publication/273607635.

Bucheton, D., \& Soulé, Y. (2009). Les gestes professionnels et le jeu des postures de l'enseignant dans la classe : un multi-agenda de préoccupations enchâssées. Éducation et didactique [En ligne], 3 (3), https://journals.openedition.org/educationdidactique/543.

Brousseau, G. (1986). Fondements et méthodes de la didactique des mathématiques, Recherche en didactique des mathématiques. Grenoble : La Pensée Sauvage.

Bruner, J. (1983). Le développement de l'enfant : savoir faire, savoir dire. Paris : Presses Universitaires de France. 
Cardin, Y. (2016). L'analyse de l'activité comme préalable à la conception d'un environnement virtuel de formation : le cas d'une formation à la gestion d'incendies en milieu urbain chez les sapeurs-pompiers. Thèse de doctorat en psychologie ergonomique, Université de Bretagne occidentale - Brest.

Clot, Y. (2001). Méthodologie en clinique de l'activité. L'exemple du sosie. In M. Santiago, \& G. Rouan (Eds.), Les méthodes qualitatives en psychologie (pp. 125-147). Paris : Dunod.

Clot, Y., Faïta, D., Fernandez, G., \& Scheller, L. (2000). Entretiens en autoconfrontation croisée : une méthode en clinique de l'activité. Perspectives interdisciplinaires sur le travail et la santé [En ligne], 2(1), http://journals.openedition.org/pistes/3833.

Delmas, P., \& St Pierre, L. (2011). La simulation, plus qu'un outil didactique, une approche pédagogique. Soins cadres, S80, 11-14.

Dreifuerst, K.T. (2012). Using debriefing for meaningful learning to foster development of clinical reasoning in simulation. Journal of Nursing Education, 51(6), 326-333.

Flin, R., \& Maran, N., (2004). Identifying and training non-technical skills for team in acute medicine, BMJ Quality and Safety, i80-i84.

Gaba, D., Howard, S., Fish, K. J., Smith, B.E., \& Sowb, Y A. (2001). Simulation-Based Training in Anesthesia Crisis Resource Management (ACRM). Simulation and Gaming, 32(2), 175-193

Glaser, B., \& Strauss, A. (1967). The Discovery of Grounded Theory: Strategies for Qualitative Research. Chicago: Aldine de Gruyter.

Granry, J.-C., \& Moll, M.C. (2012). Guide de bonnes pratiques en matière de simulation en santé. Paris : Haute Autorité de Santé.

Hetzell Campbell, S. \& Daley, K. M. (Eds) (2009). Simulation Scenarios for Nurse Educators, Making It Real. New York, NY : Springer Publishing Company.

Horcik, Z. (2014). Former des professionnels via la simulation : confrontation des principes pédagogiques issus de la littérature et des pratiques de terrain, Activités [En ligne], 11(2), https:// journals.openedition.org/activites/963.

Horcik, Z. \& Durand, M. (2011). Une démarche d'ergonomie de la formation. Activités, 8(2). https:// activites.revues.org/2613

Huber, M., \& Chautard, P. (2001). Le savoir caché des enseignants. Paris : L'Harmattan. International Nursing Association for Clinical Simulation and Learning, (2016). INACSL Standards of Best Practice: Simulation ${ }^{S M}$ Facilitation. http://www.nursingsimulation.org/article/S1876-1399 (16)30128-1/fulltext.

Jean, A., \& Etienne, R. (2009). Madame, c'est quoi un pourcentage ? Classe de $4^{\mathrm{e}}$ : La gestion des imprévus par un professeur stagiaire. In D. Bucheton (Ed.), L'agir professionnel enseignant : des gestes professionnels ajustés (pp. 97-110). Toulouse : Octarès.

Jeffries, P.R. (2005). A framework for designing, implementing, and evaluating simulations used as teaching strategies in nursing. Nurs Educ Perspect, 26(2), 96-103.

Kolb, D. A. (1984). Experiential learning: Experience as the source of learning and development (Vol. 1). Englewood Cliffs, NJ : Prentice-Hall.

Lenoir, Y., \& Pastré, P. (2008). Didactique professionnelle et didactique disciplinaires en débat. Toulouse : Octarès 
Mahoudeau, G., \& Berton, J. (2013). Séance de simulation avec un mannequin haute-fidélité. Construire un scénario. In S. Boët, J.-C. Granry, \& G. Savoldelli (Eds.), La simulation en santé : de la théorie à la pratique (pp. 289-299). Paris : Springer-Verlag.

MINISTÈRE DE LA SANTÉ ET DES SPORTS. Arrêté du 31 juillet 2009, relatif au Diplôme d'État infirmier. Récupéré de : https://www.legifrance.gouv.fr/affichTexte.do?cidTexte=JORFTEXT000020961044.

MINISTÈRE DES AFFAIRES SOCIALES ET DE LA SANTÉ. Arrêté du 26 septembre 2014, modifiant l'Arrêté du 31 juillet 2009 relatif au diplôme d'État infirmier.

Nyssen, A.-S. (2005). Simulateurs dans le domaine de l'anesthésie. Études et réflexion sur la notion de validité et de fidélité. In P. Pastré (Ed.). Apprendre par la simulation. De l'analyse du travail aux apprentissages professionnels (pp. 269-284). Toulouse : Octarès.

Paillé, P., \& Mucchielli, A., (2012). L'analyse qualitative en sciences humaines et sociales. Paris : Armand Colin ( $3^{\mathrm{e}}$ édition).

Pastré, P. (2005). Apprendre par la résolution de problèmes : le rôle de la simulation. In P. Pastré (Ed.), Apprendre par la simulation. De l'analyse du travail aux apprentissages professionnels (pp. 17-40). Toulouse : Octarès.

Pastré, P. (2011, nov.). Situations d'apprentissage et conceptualisation. Recherches en Education, 12, $12-25$.

Rabardel, P. (1995). Les hommes et les technologies; approche cognitive des instruments contemporains. Paris : Armand Colin.

Robert, A., \& Rogalski, J. (2002). Le système complexe et cohérent des pratiques des enseignants de mathématiques : une double approche. Revue canadienne de l'enseignement des sciences, des mathématiques et des technologies, 2(4), 505-528.

Rogalski, J., \& Samurçay, R. (1998). Exploitation didactique des situations de simulation. Le Travail Humain, 61(4), 333-359.

Rudolph, J.W., Simon, R., Rivard, P., Dufresne, R., \& Raemer, D.B., (2007) Debriefing with good judgment : combining rigorous feedback and genuine inquiry. Anesthesiology Clin, 25, 361-376.

Samurçay, R. (2005). Concevoir des situations simulées pour la formation professionnelle : une approche didactique. In P. Pastré (Ed..), Apprendre par la simulation : de l'analyse du travail aux apprentissages professionnels (pp. 221-240). Toulouse : Octarès,.

Savoldelli, G., \& Boët, S. (2013). Séance de simulation : du briefing au débriefing. In S. Boët, J.C. Granry, \& G. Savoldelli (Eds.), La simulation en santé : de la théorie à la pratique (pp. 313-328). Paris : Springer-Verlag.

Simoneau, I.L., \& Paquette, C. (2014). Pédagogie par la simulation clinique haute-fidélité dans la formation collégiale en santé. Rapport de recherche. CEGEP de Sherbrooke. Récupéré du site : https:// cdc.qc.ca/parea/788796-simoneau-paquette-pedagogie-simulation-clinique-formation-collegialesante-sherbrooke-PAREA-2014.pdf, consulté le 12 juillet 2016.

Vermersch, P. (1994). L'entretien d'explicitation. Paris : ESF.

Vidal-Gomel, C., Boccara, V., Rogalski, J., \& Delhomme, P. (2008). Les activités de guidage des formateurs au cours d'un audit destiné à des conducteurs expérimentés et âgés. Travail \& Apprentissage, 2, 46-64.

Vidal-Gomel, C., Fauquet-Alekhine, P., \& Guibert, S. (2011). Réflexions et apports théoriques sur la pratique des formateurs et de la simulation. In P. Fauquet-Alekhine \& N. Pehuet (Eds.), Améliorer la pratique professionnelle par la simulation (pp. 115-141). Toulouse : Octarès. 
Vygotski, L. ([1934] 1997). Pensée et Langage. Paris : Éditions La Dispute.

Wagemann, L., \& Percier, M. (1995). Contribution à l'étude de la formation à la gestion de processus continus. Le cas de l'entraînement sur simulateur machine des élèves officiers de la marine marchande. Thèse de Doctorat, Paris : EPHE.

\section{NOTES}

1. Traduction libre.

2. La formation cadre de santé prépare en un an le professionnel de santé infirmier aux fonctions de manager des services de soins et de formateur en institut de formation.

3. Par souci de préserver l'anonymat des personnes, tous les prénoms ont été changés.

4. Le score de Glasgow est un indicateur de l'état de conscience d'un patient. Il comporte trois éléments : l'ouverture des yeux, la réponse verbale et la réponse motrice.

\section{RÉSUMÉS}

La simulation clinique pleine échelle tend à devenir un outil pédagogique de premier plan dans la formation des professionnels de santé. L'activité des formateurs dans ce format est un domaine de recherche émergent. Cet article se propose d'étudier l'activité de facilitation réalisée par les formateurs au cours de situations simulées. L'objectif vise à identifier les modalités de guidage et à repérer les logiques qui les sous-tendent. Le matériau empirique a été recueilli auprès de seize formateurs exerçant en Institut de formation en soins infirmiers (Ifsi) lors d'observations en situation et d'entretiens d'autoconfrontation. Nous identifions sept modalités de guidage (étayant, didactique, productif, instrumental, normatif, prospectif, et de commodité) interdépendantes et parfois en tension. La situation simulée est ainsi considérée comme un espace d'imprévus au sein duquel le formateur doit composer avec des préoccupations entremêlées et opérer des compromis équilibrés.

Full-scale clinical simulation is becoming major educational tool for healthcare professional training. Trainers' activity in this teaching method is an emergent research area. This article studies facilitation performed by the trainers during simulated situations. The aim is to identify modalities of guidance and to target logics underlying them. The empirical corpus has been gathered with sixteen in situation trainers in nursing schools, and post situation interviews. We identified seven interdependent and occasionally contradictory guidance modalities: supporting, didactical, productive, instrument-based, normative, prospective, and of convenience. Thus, the simulated situation is considered as a space of unforeseen, within which the teacher must composed with integrated preoccupations and operate well-balanced compromises.

\section{INDEX}

Keywords : clinical simulation in healthcare, nurse training, trainer, guidance, facilitation

Mots-clés : simulation en santé, formation infirmière, formateur, guidage, facilitation 
AUTEUR

FLORENCE POLICARD

CREF, Paris Nanterre - florence.policard@gmail.com 